\title{
Robust Design for Intelligent Reflecting Surface Assisted MIMO-OFDMA Terahertz IoT Networks
}

\author{
Wanming Hao, Member, IEEE, Gangcan Sun, Ming Zeng, Member, IEEE, Zheng Chu, Member, IEEE, Zhengyu \\ Zhu, Member, IEEE, Octavia A. Dobre, Fellow, IEEE, Pei Xiao, Senior Member, IEEE,
}

\begin{abstract}
Terahertz (THz) communication has been regarded as one promising technology to enhance the transmission capacity of future internet-of-things (IoT) users due to its ultra-wide bandwidth. Nonetheless, one major obstacle that prevents the actual deployment of $\mathrm{THz}$ lies in its inherent huge attenuation. Intelligent reflecting surface (IRS) and multiple-input multipleoutput (MIMO) represent two effective solutions for compensating the large pathloss in $\mathrm{THz}$ systems. In this paper, we consider an IRS-aided multi-user THz MIMO system with orthogonal frequency division multiple access, where the sparse radio frequency chain antenna structure is adopted for reducing the power consumption. The objective is to maximize the weighted sum rate via jointly optimizing the hybrid analog/digital beamforming at the base station and reflection matrix at the IRS. Since the analog beamforming and reflection matrix need to cater all users and subcarriers, it is difficult to directly solve the formulated problem, and thus, an alternatively iterative optimization algorithm is proposed. Specifically, the analog beamforming is designed by solving a MIMO capacity maximization problem, while the digital beamforming and reflection matrix optimization are both tackled using semidefinite relaxation technique. Considering that obtaining perfect channel state information (CSI) is a challenging task in IRS-based systems, we further explore the case with the imperfect CSI for the channels from the IRS to users. Under this setup, we propose a robust beamforming and reflection matrix design scheme for the originally formulated non-convex optimization problem. Finally, simulation results are presented to demonstrate the effectiveness of the proposed algorithms.
\end{abstract}

Index Terms-Hybrid beamforming, Intelligent Reflecting Surfaces, THz, Multiple-input multiple-output.

\section{INTRODUCTION}

With the rapid proliferation of internet of things (IoTs) users, the future IoT networks need to support the huge transmission capacity [1], [2]. As such, the sub-6 Gigahertz (GHz) and millimeter-wave (mmWave) may not be able to support these users communications. That being said, terahertz $(\mathrm{THz})$ communication $(0.1-10 \mathrm{THz})$ has been regarded as a promising technology to deal with the above problem due to its ultra-wide bandwidth [3], [4]. However, there are two major shortcomings for $\mathrm{THz}$ communications, namely severe signal attenuation and poor diffraction [5].

W. Hao, G. Sun, and Z. Zhu are with the School of Information Engineering, Zhengzhou University, Zhengzhou 450001, China. (E-mail: \{iewmhao, iegcsun, iezyzhu\}@zzu.edu.cn)

M. Zeng and O. A. Dobre are with the Faculty of Engineering and Applied Science, Memorial University, St. Johns, NL A1B 3X9, Canada. (E-mail: \{mzeng, odobre\}@mun.ca)

Z. Chu and P. Xiao are with the 5G Innovation Center, Institute of Communication Systems, University of Surrey, Guildford GU2 7XH, U.K. (Email: Andrew.chuzheng7@gmail.com,p.xiao@surrey.ac.uk)
Multiple-input multiple-output (MIMO) has been recognized as an effective technology to enhance the $\mathrm{THz}$ signal strength owing to the high beamforming gain. Indeed, it has been shown that the signal strength grows linearly with the number of antennas at the base station (BS) [6]. Meanwhile, the small wavelength in $\mathrm{THz}$ makes it easy to pack more antennas together, and form a massive MIMO array. This way, the problem of severe signal antenuation of $\mathrm{THz}$ can be substantially relieved. Nonetheless, the property of poor diffraction still makes $\mathrm{THz}$ vulnerable to blocking obstacles that break the line-of-sight (LoS) links. To address this problem, intelligent reflect surface (IRS) can be deployed to create additional links [7], [8], and thus, enhance the performance of $\mathrm{THz}$ systems. Being equipped with a large number of reconfigurable passive elements [9][11], IRS can reflect the incident signals to any direction via adjusting the phase shifts. As a result, when there is no direct link between the transmitter and receiver, communication can still be realized via building a reflective link with the help of the IRS as shown in Fig. 1. Therefore, incorporating MIMO and IRS into the $\mathrm{THz}$ communication can effectively enhance the signal reception and reduce the probability of signal blockage.

In this paper, we study a multi-user IRS-aided THz MIMO system, where the BS employs sparse RF chain structure for lowering the circuit power consumption [12]. Meanwhile, considering that the wideband $\mathrm{THz}$ signals may suffer from frequency selective fading, orthogonal frequency division multiple (OFDM) is also adopted. Based on this system model, we design the hybrid analog/digital beamforming at the BS and the reflection matrix at the IRS for maximizing the weighted sum rate under perfect and imperfect channel state information (CSI).

\section{A. Related Works}

The MIMO THz communication has become a research hotspot in recent years. Considering the large signal attenuation, Lin et al. study the indoor short range MIMO $\mathrm{THz}$ communications [13], [14]. The authors propose a hybrid analog/digital beamforming to maximize the energy efficiency of the system. Busari et al. consider three hybrid beamforming array structures, namely fully-connected, subconnected and overlapped subarray [15]. Then, a single-path $\mathrm{THz}$ channel model is used to investigate the performance of the system under different array structures. Additionally, due to the ultra-wide bandwidth, frequency selective hybrid beamforming design in $\mathrm{THz}$ system is necessary. For example, Tan and Dai first analyze the array 
gain loss in the wideband $\mathrm{THz}$ system and then propose a time delay network to obtain the near-optimal array gain [16]. However, the complexity of the considered system is prohibitively high. Yuan et al. build a $3-\mathrm{D}$ wideband $\mathrm{THz}$ channel model and propose a two-stage hybrid analog/digital beamforming for maximizing the capacity of the system [17]. After that, the imperfect CSI is also considered and a robust beamforming design scheme is developed.

In parallel, IRS has attracted great attention in the past two years owning to its ability to enable cost-effective and energyefficient communications. Wu and Zhang provide a basic IRS communication system model in [9], based upon which the joint active beamforming at the BS and passive beamforming at the IRS is designed to minimize the system power consumption. In addition, Ning et al. propose to apply THz to IRS [18], and consider the beam training and hybrid analog/digital beamforming. They propose two effective hierarchical codebooks and beamforming design schemes to obtain the near-optimal performance. To study the performance of IRS in frequencyselective fading channels, Zhang et al. consider a MIMOOFDM system [19], where only one common set of IRS reflective matrix is designed for all subcarriers. Based on this, a new alternative optimization algorithm is proposed. Yang et $a l$. investigate the channel estimation and beamforming design problem in the IRS-based OFDM system [20], and propose a practical transmission protocol as well as channel estimation scheme. On this basis, a strategy of jointly optimizing power allocation and the reflection matrix is developed for maximizing the achievable rate.

Although $\mathrm{THz}$ and IRS techniques have been investigated in the literature, e.g., in [9], [13]-[21], most of them do not consider the hybrid beamforming at the BS for IRS communication [9], [18]-[21]. In fact, in a THz-based IRS communication system, the BS should employ a sparse RF antenna structure for reducing the power consumption and the multiple subcarriers transmission technology for overcoming the frequency selection channel fading. As a result, how to design the hybrid analog/digital beamforming at the BS and reflection matrix at the IRS catering to all subchannels will be challenging. In addition, how to obtain the perfect CSI remains a non-trivial task for IRS-based reflection links. For the direct link from the BS to users, the CSI can be readily estimated by conventional channel estimation methods. For the indirect link from the BS to the IRS, the CSI is also relatively easy to obtain since the locations of IRS and BS are fixed. However, the accurate CSIs of reflection links from the IRS to users are usually difficult to obtain due to the mobility of users. Nonetheless, [9], [18]-[21] all assume perfect CSI. Although Zhou et al. investigate the robust beamforming design in an IRS system [22], the conventional multiple antenna structure and single carrier scenario are considered.

\section{B. Main Contributions}

To the best of our knowledge, this is the first work to consider hybrid analog/digital beamforming in IRS-aided THz MIMO-
OFDMA under imperfect CSI, and the main contributions of this paper include:

- We construct an IRS-aided THz MIMO-OFDMA IoT communication system, where the BS employs sparse RF chain structure for reducing the circuit power consumption. On this basis, we investigate the joint optimization of the hybrid beamforming at the BS and reflection matrix at the IRS for maximizing the weighted sum rate under perfect CSI.

- To solve the formulated non-trivial problem, we first initialize the reflection matrix. Since all subcarriers share one analog beamforming matrix, we ignore the multi-user interference and obtain the analog beamforming by solving the corresponding MIMO capacity optimization problem. We subsequently reformulate a multi-user weighted sum rate maximization problem to optimize the digital beamforming. With the help of successive convex approximation (SCA) and semidefinite relaxation (SDR) techniques, we propose an iterative algorithm to solve the digital beamforming that mitigates the multi-user interference. Following this, we formulate the reflection matrix optimization problem under given hybrid analog/digital beamforming, and an iterative algorithm is proposed to solve it. The above procedure is repeated until convergence.

- Next, we assume that the perfect CSIs of reflection links cannot be obtained, and there exists bounded estimation error. We apply the same method to solve the analog beamforming. For the digital beamforming and reflection matrix, we develop a robust optimization scheme for the weighted sum rate optimization problem relying on the $\mathcal{S}$ Procedure and the convex approximation techniques. Finally, our simulation results demonstrate the effectiveness of the proposed algorithms.

We organize the rest of this paper as follows. The system model and weighted sum rate optimization problem are introduced in Section II. An alternatively iterative optimization algorithm is designed in Section III. The imperfect CSIs from the IRS to users are considered and the corresponding optimization algorithm is developed in Section IV. Simulation results are presented in Section V. Finally, conclusions are drawn in Section VI.

Notations: We use the following notations throughout this paper: $(\cdot)^{T}$ and $(\cdot)^{H}$ denote the transpose and Hermitian transpose, respectively, $\|\cdot\|$ is the Frobenius norm, $\mathbb{C}^{x \times y}$ means the space of $x \times y$ complex matrix, $\operatorname{Re}(\cdot)$ and $\operatorname{Tr}(\cdot)$ denote real number operation and trace operation, respectively, and $\operatorname{diag}\left(a_{1}, \ldots, a_{n}\right)$ is a diagonal matrix. $\angle(\cdot)$ represents the phase of a complex number.

\section{System Model and Problem Formulation}

In this section, we first describe the IRS-aided THz MIMOOFDMA system model and antenna structure. Next, we present the $\mathrm{THz}$ channel transmission model and corresponding parameters. Finally, we formulate the weighted sum rate maximization problem. 


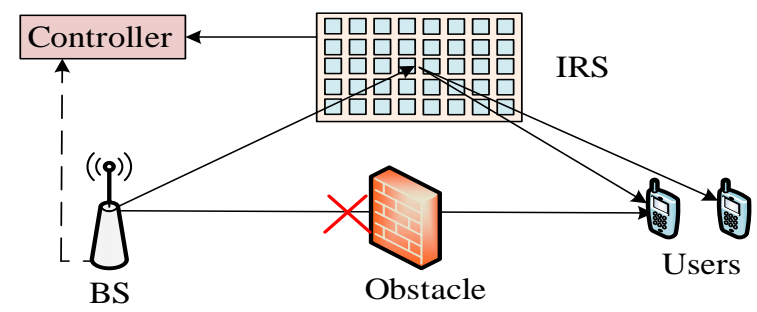

Fig. 1: The IRS system model.

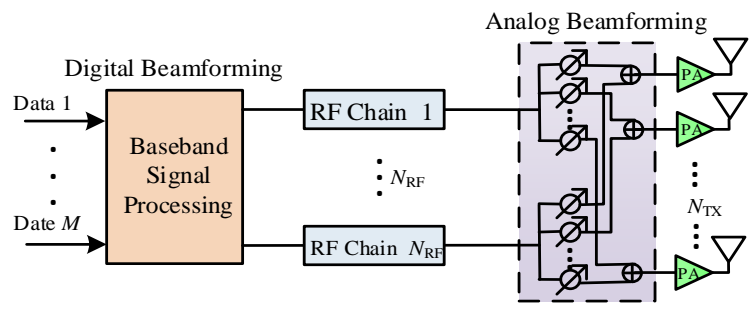

Fig. 2: The sparse RF chain structure at the BS.

\section{A. System Model}

We consider an IRS-aided THz multi-user MIMO system with OFDMA as shown in Fig. 1, where the BS is equipped with $N_{\mathrm{TX}}$ antennas and $N_{\mathrm{RF}}\left(N_{\mathrm{RF}} \leq N_{\mathrm{TX}}\right) \mathrm{RF}$ chains. The diagram of the sparse RF chain at the $\mathrm{BS}$ is illustrated in Fig. 2. We assume that there are no direct links between BS and users due to the blockage of walls or other obstacles, and the users can only receive the reflected signals from IRS. Let $N_{\text {IRS }}, M$ and $K$ denote the number of IRS elements, users and subcarriers, respectively. We assume that the CSIs of all links can be obtained using existing channel estimation schemes proposed in broadband IRS system [23]-[25]. In addition, the computation of resource allocation is executed in BS, and then the BS needs to convey the resource allocation results (refection matrix of the IRS) to IRS. As shown in Fig. 1, the IRS phased shifts are controlled by an attached controller. Therefore, the BS can transmit the reflection matrix to controller via a dedicated separate wireless control link [9].

The received signal on the $k$ th subcarrier at the $m$ th user can be expressed as

$$
y_{m}[k]=\mathbf{G}_{m}[k] \mathbf{F} \mathbf{v}_{m}[k] x_{m}[k]+\sum_{j \neq m}^{M} \mathbf{G}_{m}[k] \mathbf{F} \mathbf{v}_{j}[k] x_{j}[k]+n_{m}[k],
$$

where $\mathbf{G}_{m}[k]=G_{t} G_{r} \eta_{k} \widehat{\mathbf{g}}_{m}[k] \boldsymbol{\Phi} \widehat{\mathbf{H}}[k]$, with $G_{t}$ and $G_{r}$ as the transmit and receive antenna gains, respectively, and $\eta_{k}$ as the pathloss compensation factor [18]. $\widehat{\mathbf{g}}_{m}[k] \in \mathbb{C}^{1 \times N_{\text {IRS }}}$ denotes the channel vector from IRS to the $m$ th user on the $k$ th subcarrier, $\boldsymbol{\Phi} \in \mathbb{C}^{N_{\mathrm{IRS}} \times N_{\mathrm{IRS}}}$ is the reflection coefficient matrix with $\boldsymbol{\Phi}=\operatorname{diag}\left\{\phi_{1}, \ldots, \phi_{N_{\mathrm{IRS}}}\right\}, \widehat{\mathbf{H}}[k] \in \mathbb{C}^{N_{\mathrm{IIS}} \times N_{\mathrm{TX}}}$ represents the channel matrix from BS to IRS on the $k$ th subcarrier, $\mathbf{F} \in \mathbb{C}^{N_{\mathrm{TX}} \times N_{\mathrm{RF}}}$ is the analog beamforming matrix with $\mathbf{F}=\left[\mathbf{f}_{1}, \ldots, \mathbf{f}_{N_{\mathrm{RF}}}\right]$, $\mathbf{v}_{m}[k] \in \mathbb{C}^{N_{\mathrm{RF}} \times 1}$ and $x_{m}[k]$ denote the digital beamforming and transmit signal for the $m$ th user on the $k$ th subcarrier, respectively, $n_{m}[k]$ is the independent and identically distributed (i.i.d.) additive white Gaussian noise (AWGN) with zero-mean and variance $N_{0}$. In (1), the first term is the designed signal, while the second term is the multi-user interference that must be mitigated by designing proper digital beamforming and reflection matrix.

Next, we present the $\mathrm{THz}$ channel model. Let $f_{c}$ and $B$, respectively, represent the central frequency and bandwidth. Then, the frequency band of the $k$ th subcarrier can be expressed as $f_{k}=f_{c}+\frac{B}{K}\left(k-1-\frac{K-1}{2}\right), k=1,2, \ldots, K$. Although there are a few scattering components in $\mathrm{THz}$ communication, their power are much lower (more than $20 \mathrm{~dB}$ ) than that of LoS component [26], and thus, we only consider the LoS component and ignore the other scattering components. Accordingly, the channel matrix $\widehat{\mathbf{H}}[k]$ can be expressed as

$$
\widehat{\mathbf{H}}[k]=q\left(f_{k}, d\right) \mathbf{H}[k],
$$

where $q\left(f_{k}, d\right)$ is the complex path gain satisfying

$$
q\left(f_{k}, d\right)=\frac{c}{4 \pi f d} e^{-\frac{1}{2} \tau\left(f_{k}\right) d},
$$

where $c$ stands for the speed of light, $\tau\left(f_{k}\right)$ represents the medium absorption factor and $d$ is the distance from the BS to IRS [27]. $\mathbf{H}[k]$ can be expressed as

$$
\mathbf{H}[k]=\mathbf{a}_{r}\left(\theta_{k}\right) \mathbf{a}_{t}^{H}\left(\varphi_{k}\right),
$$

with $\mathbf{a}_{t}\left(\theta_{k}\right)$ and $\mathbf{a}_{r}\left(\varphi_{k}\right)$, respectively, as the antenna array response vector of the transmitter and receiver, namely

$$
\begin{aligned}
& \mathbf{a}_{t}\left(\theta_{k}\right)=\frac{1}{\sqrt{N_{\mathrm{TX}}}}\left[1, e^{j \pi \theta_{k}}, e^{j 2 \pi \theta_{k}}, \cdots, e^{j\left(N_{\mathrm{TX}}-1\right) \pi \theta_{k}}\right]^{T}, \\
& \mathbf{a}_{r}\left(\varphi_{k}\right)=\frac{1}{\sqrt{N_{\mathrm{IRS}}}}\left[1, e^{j \pi \varphi_{k}}, e^{j 2 \pi \varphi_{k}}, \cdots, e^{j\left(N_{\mathrm{IRS}}-1\right) \pi \varphi_{k}}\right]^{T} .
\end{aligned}
$$

Here, $\theta_{k}=2 d_{0} f_{k} \sin \left(\phi_{t}\right) / c$ and $\varphi_{k}=2 d_{0} f_{k} \sin \left(\phi_{r}\right) / c, d_{0}$ denotes the antenna distance, and $\phi_{t} / \phi_{r} \in[-\pi / 2, \pi / 2]$ are, respectively, angle of departure (AoD) and angle of arrival (AoA). Similarly, $\mathbf{g}_{m}[k]$ can be expressed as

$$
\widehat{\mathbf{g}}_{m}[k]=q\left(f_{k}, d_{m}\right) \mathbf{g}_{m}[k],
$$

where $\mathbf{g}_{m}[k]=\frac{1}{\sqrt{N_{\mathrm{IRS}}}}\left[1, e^{j \pi \varphi_{k, m}}, e^{j 2 \pi \varphi_{k, m}}, \cdots, e^{j\left(N_{\mathrm{IRS}}-1\right) \pi \varphi_{k, m}}\right]$, and $q\left(f_{k}, d_{m}\right)$ is defined as

$$
q\left(f_{k}, d_{m}\right)=\frac{c}{4 \pi f_{k} d_{m}} e^{-\frac{1}{2} \tau\left(f_{k}\right) d_{m}},
$$

with $d_{m}$ as the distance from the IRS to the $m$ th user. The BS-IRS-user $m$ link channel can such be expressed as

$$
\mathbf{G}_{m}[k]=u_{m}[k] \mathbf{g}_{m}[k] \mathbf{\Phi} \mathbf{H}[k],
$$

where $u_{m}[k]=G_{t} G_{r} \eta_{k} q\left(f, d_{m}\right) q\left(f_{k}, d_{m}\right)$. According to [27], the cascaded path loss of the BS-IRS-user link should satisfy

$$
\eta_{k} q\left(f_{k}, d\right) q\left(f_{k}, d_{m}\right)=\frac{\chi c}{8 \sqrt{\pi^{3}} f_{k} d d_{m}} e^{-\frac{1}{2} \tau\left(f_{k}\right)\left(d+d_{m}\right)},
$$

where $\chi$ is the IRS element gain.

Finally, we rewrite (1) as

$$
\begin{aligned}
y_{m}[k] & =u_{m}[k] \mathbf{g}_{m}[k] \mathbf{\Phi} \mathbf{H}[k] \mathbf{F} \mathbf{v}_{m}[k] x_{m}[k] \\
& +\sum_{j \neq m}^{M} u_{m}[k] \mathbf{g}_{m}[k] \mathbf{\Phi} \mathbf{H}[k] \mathbf{F} \mathbf{v}_{j}[k] x_{j}[k]+n_{m}[k] .
\end{aligned}
$$




\section{B. Problem Formulation}

By employing (10), the achievable rate of the $m$ th user on the $k$ th subcarrier can be expressed as

$R_{m}[k]=\frac{B}{K} \log \left(1+\frac{\left|u_{m}[k] \mathbf{g}_{m}[k] \mathbf{\Phi} \mathbf{H}[k] \mathbf{F} \mathbf{v}_{m}[k]\right|^{2}}{\sum_{j \neq m}^{M}\left|u_{m}[k] \mathbf{g}_{m}[k] \mathbf{\Phi} \mathbf{H}[k] \mathbf{F} \mathbf{v}_{j}[k]\right|^{2}+B N_{0} / K}\right)$,

and thus, the achievable sum rate for the $m$ th user can be written as

$$
R_{m}=\sum_{k=1}^{K} R_{m}[k] .
$$

Next, we formulate the weighted sum rate maximization problem as follows

$$
\begin{aligned}
& \max _{\left\{\mathbf{\Phi}, \mathbf{F}, \mathbf{v}_{m}[k]\right\}} \sum_{m=1}^{M} \alpha_{m} R_{m} \\
& \text { s.t. }\left|\phi_{i}\right|=1, i \in\left\{1, \cdots, N_{\mathrm{IRS}}\right\}, \\
& \quad \sum_{M=1}^{M} \sum_{k=1}^{K}\left\|\mathbf{F} \mathbf{v}_{m}[k]\right\|^{2} \leq P_{\max }, \\
& \quad \mathbf{F}(i, j)=1 / \sqrt{N_{\mathrm{TX}}}, i \in\left\{1, \cdots, N_{\mathrm{TX}}\right\}, j \in\left\{1, \cdots, N_{\mathrm{RF}}\right\},
\end{aligned}
$$

where $\alpha_{m}$ denotes the weight of the $m$ th user, (13b) is the uni-modular constraint for each reflection coefficient $\phi_{i},(13 \mathrm{c})$ is the sum transmit power constraint, and (13d) is the amplitude constraint of analog beamforming. The objective of (13) is to jointly optimize the reflection matrix $\boldsymbol{\Phi}$ and hybrid analog/digital beamforming $\mathbf{F}$ and $\mathbf{v}_{m}[k]$ for maximizing the weighed sum rate of the system. Obviously, (13) is a nonconvex optimization problem due to the non-convex objective function (13a) and constraints (13b), (13d). We need to optimize the reflect matrix $\boldsymbol{\Phi}$, analog beamforming $\mathbf{F}$, and digital beamforming $\mathbf{v}_{m}[k]$ for the original problem. Obviously, reflect matrix $\boldsymbol{\Phi}$ and analog beamforming $\mathbf{F}$ need to cater for all subcarriers as [28]. Finding the optimal solution is a challenging task, and we propose an effective alternatively iterative optimization algorithm to deal with it.

\section{Solution of The Weighted Sum Rate Optimization Problem}

In this section, we propose an alternatively iterative optimization algorithm. First, we consider the hybrid analog/digital beamforming of $\mathbf{F}, \mathbf{v}_{m}[k]$ under given reflection matrix $\boldsymbol{\Phi}$. We formulate a MIMO-OFDM sum rate maximization problem and use it to obtain the analog beamforming matrix F. Next, we transform the original problem into a SDP and solve the digital beamforming $\mathbf{v}_{m}[k]$ by the SDR technique. Lastly, we solve the reflection matrix $\boldsymbol{\Phi}$ according to the obtained hybrid beamforming $\mathbf{F}$ and $\mathbf{v}_{m}[k]$. The above procedure is repeated until convergence.

\section{A. Optimization of $\mathbf{F}$ and $\mathbf{v}_{m}[k]$ under Fixed $\mathbf{\Phi}$}

Under given $\boldsymbol{\Phi}$, the original problem can be transformed as

$$
\max _{\left\{\mathbf{F}, \mathbf{v}_{m}[k]\right\}} \sum_{m=1}^{M} \sum_{k=1}^{K} a_{m} \log \left(1+\frac{\left|\hat{\mathbf{h}}_{m}[k] \mathbf{F} \mathbf{v}_{m}[k]\right|^{2}}{\sum_{j \neq m}^{M}\left|\hat{\mathbf{h}}_{m}[k] \mathbf{F} \mathbf{v}_{j}[k]\right|^{2}+\delta^{2}}\right)
$$

s.t. $(13 \mathrm{c}),(13 \mathrm{~d})$, where $\hat{\mathbf{h}}_{m}[k]=u_{m}[k] \mathbf{g}_{m}[k] \mathbf{\Phi H}[k], a_{m}=B \alpha_{m} / K$, and $\delta^{2}=$ $B N_{0} / K$.

Problem (14) is still difficult to solve since each common element of the analog beamforming $\mathbf{F}$ needs to cater for all users and subcarriers. Furthermore, the data streams of different users may own different priority weights, which leads to more complicated analog beamforming design. For simplicity, we assume that all users have the same weights and it can be regarded as a MIMO-OFDM system by neglecting the interuser interference. To solve the analog beamforming $\mathbf{F}$, we first define $\hat{\mathbf{H}}[k]=\left[\hat{\mathbf{h}}_{1}[k]^{T}, \ldots, \hat{\mathbf{h}}_{M}[k]^{T}\right]^{T}$, and reformulate a MIMOOFDM sum rate maximization problem as follows:

$$
\begin{aligned}
& \max _{\{\mathbf{F}, \mathbf{V}[k]\}} \frac{1}{K} \sum_{k=1}^{K} \log \left(\mathbf{I}+\frac{\hat{\mathbf{H}}[k] \mathbf{F V}[k] \mathbf{V}[k]^{H} \mathbf{F}^{H} \hat{\mathbf{H}}[k]^{H}}{\delta^{2}}\right) \\
& \text { s.t. }\|\mathbf{F V}[k]\|^{2} \leq P_{\max } / K,(13 \mathrm{~d}) .
\end{aligned}
$$

Here, we consider the transmit power constraint for each subcarrier to obtain the lower bound. Note that (15) is only used for designing $\mathbf{F}$. For a given $\mathbf{F}$, the optimal digital beamforming matrix can be calculated as [29]

$$
\mathbf{V}[k]=\left(\mathbf{F}^{H} \mathbf{F}\right)^{-1 / 2} \mathbf{U}_{e}[k] \boldsymbol{\Gamma}_{e}[k],
$$

where $\mathbf{U}_{e}[k]$ is the set of right singular vector according to the $N_{\mathrm{RF}}$ largest singular value of $\hat{\mathbf{H}}[k] \mathbf{F}\left(\mathbf{F}^{H} \mathbf{F}\right)^{-1 / 2}$, and $\boldsymbol{\Gamma}_{e}[k]$ is a diagonal matrix of the power allocated to the data streams on each subcarrier. Here, we assume that there are $N_{\mathrm{RF}}$ data streams. In addition, it is obvious that $\mathbf{f}_{i}^{H} \mathbf{f}_{i}=1$, while $\mathbf{f}_{i}^{H} \mathbf{f}_{j} \ll 1(i \neq j)$ with high probability for a large $N_{\mathrm{TX}}$. Therefore, for the i.i.d. channels, the analog beamforming satisfying $\mathbf{F}^{H} \mathbf{F} \approx \mathbf{I}$ can always be approximated as proportional to the identity matrix, namely $\mathbf{F}^{H} \mathbf{F} \propto \mathbf{I}$. Moreover, in the high or moderate signal-tonoise ratio (SNR) regime, the equal power allocation scheme for all streams on each subcarrier can be adopted without significant performance degradation, namely $\boldsymbol{\Gamma}_{e}[k] \propto \mathbf{I}[30]$. As a result, the digital beamforming matrix can be approximated as $\mathbf{V}[k] \approx \lambda \mathbf{U}_{e}[k]$, where $\lambda=\sqrt{P_{\max } /\left(K N_{\mathrm{TX}} N_{\mathrm{RF}}\right)}$. Based on the above analysis, we have

$$
\begin{aligned}
& \frac{1}{K} \sum_{k=1}^{K} \log \left(\mathbf{I}+\frac{\hat{\mathbf{H}}[k] \mathbf{F V}[k] \mathbf{V}[k]^{H} \mathbf{F}^{H} \hat{\mathbf{H}}[k]^{H}}{\delta^{2}}\right) \\
= & \frac{1}{K} \sum_{k=1}^{K} \log \left(\mathbf{I}+\frac{\lambda}{\delta^{2}} \hat{\mathbf{H}}[k] \mathbf{F} \mathbf{U}_{e}[k] \mathbf{U}_{e}[k]^{H} \mathbf{F}^{H} \hat{\mathbf{H}}[k]^{H}\right) \\
= & \frac{1}{K} \sum_{k=1}^{K} \log \left(\mathbf{I}+\frac{\lambda}{\delta^{2}} \mathbf{F}^{H} \hat{\mathbf{H}}[k]^{H} \hat{\mathbf{H}}[k] \mathbf{F}\right) .
\end{aligned}
$$

Finally, we can obtain the upper bound of (17) using the Jensen's inequality ${ }^{1}$ as

$$
\frac{1}{K} \sum_{k=1}^{K} \log \left(\mathbf{I}+\frac{\lambda}{\delta^{2}} \mathbf{F}^{H} \hat{\mathbf{H}}[k]^{H} \hat{\mathbf{H}}[k] \mathbf{F}\right) \leq \log \left(\mathbf{I}+\frac{\lambda}{\delta^{2}} \mathbf{F}^{H} \mathbf{\Sigma} \mathbf{F}\right),
$$

\footnotetext{
${ }^{1}$ The Jensen's inequality is defied as: If $f(x), x \in[a, b]$ is a convex function, and then for any $x_{1}, x_{2}, \cdots, x_{n} \in[a, b]$, we have $\frac{\sum_{i=1}^{n} f\left(x_{i}\right)}{n} \leq f\left(\frac{\sum_{i=1}^{n} x_{i}}{n}\right)$ and the equality holds only when $x_{1}=x_{2}=\cdots=x_{n}$.
} 
where $\boldsymbol{\Sigma}=\frac{1}{K} \sum_{k=1}^{K}\left(\hat{\mathbf{H}}[k]^{H} \hat{\mathbf{H}}[k]\right)$ and the analog beamforming matrix can be obtained by solving the following problem

$$
\mathbf{F}^{\star}=\underset{\mathbf{F}(i, j)=1 / \sqrt{N_{\mathrm{TX}}}}{\arg \max } \log \left(\mathbf{I}+\frac{\lambda}{\delta^{2}} \mathbf{F}^{H} \mathbf{\Sigma} \mathbf{F}\right) .
$$

Since $\boldsymbol{\Sigma}$ is a hermitian matrix, its singular value decomposition (SVD) can be written as $\boldsymbol{\Sigma}=\mathbf{S} \boldsymbol{\Lambda} \mathbf{S}^{H}$. Therefore, the solution of (19) can be given by $\mathbf{F}^{\star}(i, j)=\frac{1}{\sqrt{\sqrt{T X}}} e^{\left\langle\left(\mathbf{S}_{1: N_{\mathrm{BF}}}(i, j)\right)\right.}$, where $\mathbf{S}_{1: N_{\mathrm{BF}}}$ denotes the first $N_{\mathrm{BF}}$ columns of $\mathbf{S}$.

Note that although (16) provides a digital beamforming solution, it is only suitable for the single user case. Therefore, after obtaining the analog beamforming matrix $\mathbf{F}$, problem (14) can be transformed as follows for solving digital beamforming

$$
\begin{aligned}
& \max _{\left\{\mathbf{v}_{m}[k]\right\}} \sum_{m=1}^{M} \sum_{k=1}^{K} a_{m} \log \left(1+\frac{\left|\overline{\mathbf{h}}_{m}[k] \mathbf{v}_{m}[k]\right|^{2}}{\sum_{j \neq m}^{M}\left|\overline{\mathbf{h}}_{m}[k] \mathbf{v}_{j}[k]\right|^{2}+\delta^{2}}\right) \\
\text { s.t. } & \sum_{M=1}^{M} \sum_{k=1}^{K}\left\|\mathbf{F} \mathbf{v}_{m}[k]\right\|^{2} \leq P_{\max },
\end{aligned}
$$

where $\overline{\mathbf{h}}_{m}[k]=\hat{\mathbf{h}}_{m}[k] \mathbf{F}$. We define $\overline{\mathbf{H}}_{m}[k]=\overline{\mathbf{h}}_{m}[k]^{H} \overline{\mathbf{h}}_{m}[k]$ and $\mathbf{V}_{m}[k]=\mathbf{v}_{m}[k] \mathbf{v}_{m}[k]^{H}$, and by using an auxiliary variable $t_{m, k}$, we reformulate (20) as

$$
\begin{aligned}
& \max _{\left\{\mathbf{V}_{m}[k]\right\}} \sum_{m=1}^{M} \sum_{k=1}^{K} a_{m} \log \left(1+t_{m, k}\right) \\
\text { s.t. } & t_{m, k} \leq \frac{\operatorname{Tr}\left(\overline{\mathbf{H}}_{m}[k] \mathbf{V}_{m}[k]\right)}{\sum_{j \neq m}^{M} \operatorname{Tr}\left(\overline{\mathbf{H}}_{m}[k] \mathbf{V}_{j}[k]\right)+\delta^{2}}, \\
& \sum_{M=1}^{M} \sum_{k=1}^{K} \operatorname{Tr}\left(\mathbf{F}^{H} \mathbf{F} \mathbf{V}_{m}[k]\right) \leq P_{\max }, \\
& \operatorname{rank}\left(\mathbf{V}_{m}[k]\right)=1, \mathbf{V}_{m}[k] \geq 0 .
\end{aligned}
$$

It is obvious that (21) is a non-convex optimization problem due to (21b) and (21d). To cope with (21b), we introduce an auxiliary variable $b_{m, k}$ and transform it as

$$
\begin{aligned}
& t_{m, k} b_{m, k} \leq \operatorname{Tr}\left(\overline{\mathbf{H}}_{m}[k] \mathbf{V}_{m}[k]\right), \\
& b_{m, k} \geq \sum_{j \neq m}^{M} \operatorname{Tr}\left(\overline{\mathbf{H}}_{m}[k] \mathbf{V}_{j}[k]\right)+\delta^{2} .
\end{aligned}
$$

Now, we only need to deal with $t_{m, k} b_{m, k}$. According to [31], the upper bound of $t_{m, k} b_{m, k}$ can be obtained as

$$
\frac{t_{m, k}^{(n)}}{2 b_{m, k}^{(n)}} b_{m, k}^{2}+\frac{b_{m, k}^{(n)}}{2 t_{m, k}^{(n)}} t_{m, k}^{2} \geq t_{m, k} b_{m, k},
$$

where $t_{m, k}^{(n)}$ and $b_{m, k}^{(n)}$ are the values of $t_{m, k}$ and $b_{m, k}$ at the $n$th iteration, respectively. Consequently, we transform (22a) into the following convex constraints

$$
\frac{t_{m, k}^{(n)}}{2 b_{m, k}^{(n)}} b_{m, k}^{2}+\frac{b_{m, k}^{(n)}}{2 t_{m, k}^{(n)}} t_{m, k}^{2} \leq \operatorname{Tr}\left(\overline{\mathbf{H}}_{m}[k] \mathbf{V}_{m}[k]\right) .
$$

Finally, (21) can be recast as the following SDP problem

$$
\begin{aligned}
& \max _{\left\{t_{m, k}, b_{m, k}, \mathbf{V}_{m}[k]\right\}} \sum_{m=1}^{M} \sum_{k=1}^{K} a_{m} \log \left(1+t_{m, k}\right) \\
& \text { s.t. }(21 \mathrm{c}),(22 \mathrm{~b}),(24), \\
& \operatorname{rank}\left(\mathbf{V}_{m}[k]\right)=1, \mathbf{V}_{m}[k] \geq 0 .
\end{aligned}
$$

Since the rank-one constraint is non-convex, we need to drop it and formulate a SDR problem that can be solved by existing convex solvers such as the CVX toolbox. Summarily, to obtain the digital beamforming matrix $\mathbf{V}_{m}[k]$, we need to iteratively solve (25). Specifically, we first initialize the auxiliary variables $b_{m, k}^{\mathrm{o}}, t_{m, k}^{\mathrm{o}}$ and solve (25) for obtaining the optimal $b_{m, k}^{\star}, t_{m, k}^{\star}$ and $\mathbf{V}_{m}[k]^{\star}$. Next, $b_{m, k}^{o}$ and $t_{m, k}^{0}$ are updated with the obtained $b_{m, k}^{\star}$ and $t_{m, k}^{\star}$, and then, we resolve (25). The above procedure is repeated until the results converge or the iteration number reaches its maximum value. In addition, since the SDR problem of (25) is a convex optimization problem, the solutions are optimal for each iteration. Therefore, iteratively solving (25) and updating variables increase or at least maintain the value of the objective function [32], [33]. Given the limited transmit power, the designed iterative algorithm guarantees the value of the objective function to be a monotonically non-decreasing sequence with an upper bound, and it converges to a stationary solution that is at least a local optimal.

For solving (25), we remove the rank-one constraint $\operatorname{rank}\left(\mathbf{V}_{m}[k]\right)=1$. To explore the characteristic of the solutions, we first give the following theorem for the obtained digital beamforming $\mathbf{V}_{m}^{\star}[k]$.

Theorem 1: For a large number of BS antenna $N_{\mathrm{TX}}$, the obtained digital beamforming $\mathbf{V}_{m}^{\star}[k]$ satisfies $\operatorname{rank}\left(\mathbf{V}_{m}^{\star}[k]\right)=1$.

\section{Proof Refer to Appendix A.}

In fact, even for a medium number of BS antennas, such as $N_{\mathrm{TX}}=16$, we find that the optimal $\mathbf{V}_{m}^{\star}[k]$ always satisfies $\operatorname{rank}\left(\mathbf{V}_{m}^{\star}[k]\right)=1$. If $\operatorname{rank}\left(\mathbf{V}_{m}^{\star}[k]\right)=1$, the optimal $\mathbf{V}_{m}^{\star}[k]$ can be recovered by the eigenvalue decomposition, namely $\mathbf{V}_{m}^{\star}[k]=$ $\mathbf{v}_{m}^{\star}[k]^{H} \mathbf{v}_{m}^{\star}[k]$. If the obtained $\mathbf{V}_{m}^{\star}[k]$ is not a rank-one matrix, the Gaussian randomization technique is typically used to obtain a rank-one solution [34]. Specifically, we first generate a random vector $\hat{\mathbf{v}}_{m}[k]$ satisfying $\hat{\mathbf{v}}_{m}[k] \sim C \mathcal{N}\left(\mathbf{0}, \mathbf{V}_{m}^{\star}[k]\right)$. Then, we apply $\hat{\mathbf{v}}_{m}[k]$ to problem (25) and check its feasibility. Here, we need to independently generate sufficient feasible beamforming vectors $\hat{\mathbf{v}}_{m}[k]$ and select the optimal one $\hat{\mathbf{v}}_{m}[k]^{\star}$ from all the random vectors. Based on this, the final digital beamforming vector can be approximated as $\mathbf{v}_{m}[k]^{\star}=\hat{\mathbf{v}}_{m}[k]^{\star}$.

Now, we analyze the computational complexity for solving problem (25). Let $\epsilon$ be the iteration accuracy; then, the number of iterations is on the order of $\sqrt{K M N_{\mathrm{RF}}+5 K M+1} \ln (1 / \epsilon)$ [35]. For problem (25), there are $K M$ linear matrix inequality (LMI) constraints of size $N_{\mathrm{RF}}$, $3 K M+1$ LMI constraints of size 1 , and $K M$ second-order cone constraints. Therefore, the complexity of solving problem (25) is on the order of $\sqrt{\Delta_{1}} \zeta \ln (1 / \epsilon)\left(\Delta_{2}+\zeta \Delta_{3}+\zeta^{2}\right)$, where $\Delta_{1}=K M N_{\mathrm{RF}}+5 K M+1, \Delta_{2}=K M N_{\mathrm{RF}}^{3}+12 K M+1$, $\Delta_{3}=K M N_{\mathrm{RF}}^{2}+3 K M+1$, and $\zeta=K M N_{\mathrm{RF}}^{2}$.

\section{B. Optimization of $\boldsymbol{\Phi}$ under Fixed $\mathbf{F}$ and $\mathbf{V}_{m}[k]$}

After obtaining the hybrid analog/digital beamforming $\mathbf{F}$ and $\mathbf{v}_{m}[k]$, we consider the reflection matrix of IRS here and 
transform (13) into the following optimization problem

$$
\max _{\{\boldsymbol{\Phi}\}} \sum_{m=1}^{M} \sum_{k=1}^{K} a_{m} \log \left(1+\frac{\left|\mathbf{g}_{m}[k] \mathbf{\Phi} \mathbf{z}_{m}[k]\right|^{2}}{\sum_{j \neq m}^{M}\left|\mathbf{g}_{m}[k] \mathbf{\Phi} \mathbf{z}_{j}[k]\right|^{2}+\delta^{2}}\right)
$$

s.t. $\left|\phi_{i}\right|=1, i \in\left\{1, \cdots, N_{\mathrm{IRS}}\right\}$,

where $\mathbf{z}_{m}[k]=u_{m}[k] \mathbf{H}[k] \mathbf{F} \mathbf{v}_{m}[k]$. Let $\mathbf{g}_{m}[k] \mathbf{\Phi} \mathbf{z}_{m}[k]=\mathbf{c}_{m, k} \boldsymbol{\phi}$, where $\boldsymbol{\phi}=\left[\phi_{1}, \ldots, \phi_{\left.N_{\mathrm{IR}}\right]}\right]^{T}$ and $\mathbf{c}_{m}[k]=\mathbf{g}_{m}[k] \operatorname{diag}\left(\mathbf{z}_{m}[k]\right)$. Thus, we can reformulate (26) as

$$
\begin{aligned}
& \max _{\{\phi\}} \sum_{m=1}^{M} \sum_{k=1}^{K} a_{m} \log \left(1+\frac{\left|\mathbf{c}_{m}[k] \boldsymbol{\phi}\right|^{2}}{\sum_{j \neq m}^{M}\left|\mathbf{c}_{j}[k] \boldsymbol{\phi}\right|^{2}+\delta^{2}}\right) \\
& \text { s.t. }\left|\phi_{i}\right|=1, i \in\left\{1, \cdots, N_{\mathrm{IRS}}\right\} .
\end{aligned}
$$

Similarly, we define $\mathbf{C}_{m}[k]=\mathbf{c}_{m}[k]^{H} \mathbf{c}_{m}[k]$ and $\boldsymbol{\Omega}=\boldsymbol{\phi} \boldsymbol{\phi}^{H}$, and transform (27) into the following optimization problem

$$
\begin{aligned}
\max _{\{\mathbf{\Omega}\}} & \sum_{m=1}^{M} \sum_{k=1}^{K} a_{m} \log \left(1+\frac{\operatorname{Tr}\left(\mathbf{C}_{m}[k] \mathbf{\Omega}\right)}{\sum_{j \neq m}^{M} \operatorname{Tr}\left(\mathbf{C}_{j}[k] \mathbf{\Omega}\right)+\delta^{2}}\right) \\
\text { s.t. } & \boldsymbol{\Omega}(i, i)=1, i \in\left\{1, \cdots, N_{\mathrm{IRS}}\right\}, \\
& \operatorname{rank}(\boldsymbol{\Omega})=1, \boldsymbol{\Omega} \geq 0 .
\end{aligned}
$$

One can observe that (28) has a similar form to (21), and we can adopt the same iterative method proposed in Section. III-A to solve it. We omit the details due to the space limitation and directly write the SDR problem as follows:

$$
\begin{aligned}
& \max _{\left\{t_{m, k}^{\prime}, b_{m, k}^{\prime}, \mathbf{\Omega}\right\}} \sum_{m=1}^{M} \sum_{k=1}^{K} a_{m} \log \left(1+t_{m, k}^{\prime}\right) \\
& \text { s.t. } b_{m, k}^{\prime} \geq \sum_{j \neq m}^{M} \operatorname{Tr}\left(\mathbf{C}_{j}[k] \mathbf{\Omega}\right)+\delta^{2} \text {, } \\
& \frac{{t^{\prime}}_{m, k}^{(n)}}{2{b^{\prime}}_{m, k}^{(n)}}{b^{\prime 2}}_{m, k}^{2}+\frac{{b^{\prime}}_{m, k}^{(n)}}{2{t^{\prime}}_{m, k}^{(n)}}{t^{\prime 2}}_{m, k}^{2} \leq \operatorname{Tr}\left(\mathbf{C}_{m}[k] \boldsymbol{\Omega}\right), \\
& \boldsymbol{\Omega}(i, i)=1, \boldsymbol{\Omega} \geq 0 .
\end{aligned}
$$

Here, we still need to alternatively solve problem (29) to obtain the reflection matrix $\boldsymbol{\Phi}$. If the obtained $\phi^{\star}$ satisfies $\operatorname{rank}\left(\boldsymbol{\Omega}^{\star}\right)=1$, the optimal $\boldsymbol{\phi}^{\star}$ can be recovered by the eigenvector of $\boldsymbol{\Omega}^{\star}=\boldsymbol{\phi}^{\star} \boldsymbol{\phi}^{\star H}$, and the reflection coefficient matrix $\boldsymbol{\Phi}^{\star}$ can be expressed as $\boldsymbol{\Phi}^{\star}=\operatorname{diag}\left\{\phi_{1}^{\star}, \ldots, \phi_{N_{\mathrm{IRS}}}^{\star}\right\}$. Otherwise, the Gaussian randomization technique can be used to obtain a rank-one solution [34].

Now, we analyze the computational complexity for solving problem (29). Let $\epsilon$ be the iteration accuracy; then, the number of iterations is on the order of $\sqrt{2 N_{\text {IRS }}+5 K M} \ln (1 / \epsilon)$. For problem (29), there are 1 LMI constraint of size $N_{\text {IRS }}$, $3 K M+N_{\text {IRS }}$ LMI constraint of size 1 , and $K M$ secondorder cone constraints. Therefore, the complexity of solving problem (29) is on the order of $\sqrt{\Delta_{1}} \zeta \ln (1 / \epsilon)\left(\Delta_{2}+\zeta \Delta_{3}+\zeta^{2}\right)$, where $\Delta_{1}=2 N_{\text {IRS }}+5 K M, \Delta_{2}=N_{\text {IRS }}^{3}+N_{\text {IRS }}+12 K M$, $\Delta_{3}=N_{\mathrm{IRS}}^{2}+N_{\mathrm{IRS}}+3 K M$, and $\zeta=N_{\mathrm{IRS}}^{2}$.

Finally, we summarize the proposed alteratively iterative optimization scheme in Algorithm 1.

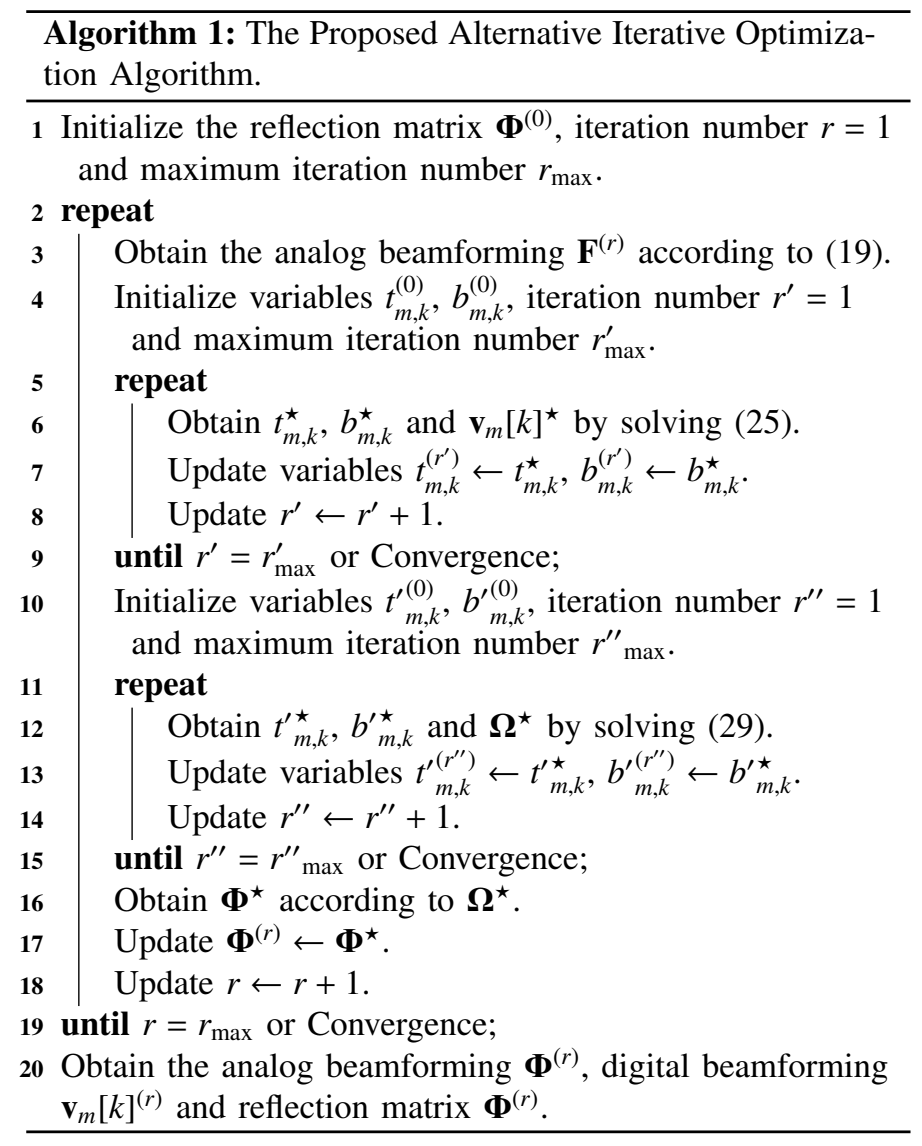

\section{EXtension to ImPerfect CSIs From IRS to Users}

Due to the mobility of users, it is difficult to obtain the perfect CSIs from the IRS to users. Therefore, in this section, we assume that there exists channel estimation error for the reflection links between IRS and users, namely $\mathbf{g}_{m}[k]=\tilde{\mathbf{g}}_{m}[k]+\Delta \mathbf{g}_{m}[k]$, where $\tilde{\mathbf{g}}_{m}[k]$ denotes the estimated CSI and $\Delta \mathbf{g}_{m}[k]$ is the estimation error. Here, we assume that the estimation error is upper bounded with $\tilde{\mathbf{g}}_{m}[k] \tilde{\mathbf{g}}_{m}[k]^{H} \leq \varepsilon$. Next, we redesign the hybrid analog/digital beamforming and reflection matrix with imperfect CSI as follows

$$
\begin{aligned}
& \max _{\left\{\boldsymbol{\Phi}, \mathbf{F}, \mathbf{v}_{m}[k]\right\}} \sum_{m=1}^{M} \alpha_{m} R_{m} \\
& \text { s.t. }\left|\phi_{i}\right|=1, i \in\left\{1, \cdots, N_{\mathrm{IRS}}\right\}, \\
& \quad \sum_{M=1}^{M} \sum_{k=1}^{K}\left\|\mathbf{F} \mathbf{v}_{m}[k]\right\|^{2} \leq P_{\max }, \\
& \quad \mathbf{g}_{m}[k]=\tilde{\mathbf{g}}_{m}[k]+\Delta \mathbf{g}_{m}[k], \\
& \tilde{\mathbf{g}}_{m}[k] \tilde{\mathbf{g}}_{m}[k]^{H} \leq \varepsilon, \\
& \quad \mathbf{F}(i, j)=1 / \sqrt{N_{\mathrm{TX}}}, i \in\left\{1, \cdots, N_{\mathrm{TX}}\right\}, j \in\left\{1, \cdots, N_{\mathrm{RF}}\right\} .
\end{aligned}
$$

Similar to the proposed Algorithm 1, we design an alternative iterative optimization scheme to solve it.

\section{A. Optimization of $\mathbf{F}$ and $\mathbf{v}_{m}[k]$ under Fixed $\mathbf{\Phi}$}

In fact, it is extremely difficult to design analog beamforming F under channel estimation error, and thus, we ignore the estimation error and define $\mathbf{g}_{m}[k] \triangleq \tilde{\mathbf{g}}_{m}[k]$ for simplicity, namely 
$\hat{\mathbf{h}}_{m}[k]=u_{m}[k] \tilde{\mathbf{g}}_{m}[k] \boldsymbol{\Phi H}[k]$. In this way, we can adopt the same scheme used in Section III-A to obtain the analog beamforming F. Next, we directly solve the digital beamforming $\mathbf{v}_{m}[k]$. After obtaining $\mathbf{F}$, we define $u_{m}[k] \mathbf{g}_{m}[k] \boldsymbol{\Phi} \mathbf{H}[k] \mathbf{F} \triangleq \mathbf{g}_{m}[k] \boldsymbol{\Xi}_{m}[k]$, where $\boldsymbol{\Xi}_{m}[k]=u_{m}[k] \boldsymbol{\Phi} \mathbf{H}[k] \mathbf{F}$. Thus, the received signal of the $m$ th user on the $k$ th subcarrier can be expressed as

$$
\begin{aligned}
y_{m}[k] & =\left(\tilde{\mathbf{g}}_{m}[k]+\Delta \mathbf{g}_{m}[k]\right) \boldsymbol{\Xi}_{m}[k] \mathbf{v}_{m}[k] x_{m}[k] \\
& +\sum_{j \neq m}^{M}\left(\tilde{\mathbf{g}}_{m}[k]+\Delta \mathbf{g}_{m}[k]\right) \boldsymbol{\Xi}_{m}[k] \mathbf{v}_{j}[k] x_{j}[k]+n_{m}[k],
\end{aligned}
$$

and the achievable rate is given by

$$
R_{m}[k]=\frac{B}{K} \log \left(1+\frac{\left|\left(\tilde{\mathbf{g}}_{m}[k]+\Delta \mathbf{g}_{m}[k]\right) \mathbf{\Xi}_{m}[k] \mathbf{v}_{m}[k]\right|^{2}}{\sum_{j \neq m}^{M} \Upsilon_{m}[k]+B N_{0} / K}\right),
$$

where $\Upsilon_{m}[k]=\left|\left(\tilde{\mathbf{g}}_{m}[k]+\Delta \mathbf{g}_{m}[k]\right) \boldsymbol{\Xi}_{m}[k] \mathbf{v}_{j}[k]\right|^{2}$. By introducing an auxiliary variable $\tau_{m, k}$, the original problem (30) can be recast as

$$
\begin{gathered}
\max _{\left\{\mathbf{v}_{m}[k]\right\}} \sum_{m=1}^{M} \sum_{k=1}^{K} a_{m} \log \left(1+\tau_{m, k}\right) \\
\text { s.t. } \tau_{m, k} \leq \frac{\left|\left(\tilde{\mathbf{g}}_{m}[k]+\Delta \mathbf{g}_{m}[k]\right) \mathbf{\Xi}_{m}[k] \mathbf{v}_{m}[k]\right|^{2}}{\sum_{j \neq m}^{M} \Upsilon_{m}[k]+B N_{0} / K}, \\
\Delta \mathbf{g}_{m}[k] \Delta \mathbf{g}_{m}[k]^{H} \leq \varepsilon, \\
\sum_{M=1}^{M} \sum_{k=1}^{K}\left\|\mathbf{F} \mathbf{v}_{m}[k]\right\|^{2} \leq P_{\max } .
\end{gathered}
$$

By comparing the weighted sum rate maximization problem under perfect CSI (21) and imperfect CSI (33), one can observe that the original scheme used to solve (21) can not be directly used to solve (33) due to the uncertain term $\Delta \mathbf{g}_{m}[k]$. To cope with (33), we first give the following Lemma 1 referred to as $\mathcal{S}$-Procedure [36].

Lemma 1: Define the function

$$
f_{i}(\mathbf{x})=\mathbf{x} \mathbf{Q}_{i} \mathbf{x}^{H}+2 \operatorname{Re}\left\{\mathbf{p}_{i} \mathbf{x}^{H}\right\}+e_{i}, i \in\{1,2\},
$$

where $\mathbf{x} \in \mathbb{C}^{1 \times O}, \mathbf{Q}_{i} \in \mathbb{C}^{O \times O}, \mathbf{p}_{i} \in \mathbb{C}^{1 \times O}$, and $e_{i} \in \mathbb{R}$ with $O$ representing any integer, and thus, the expression $f_{1}(\mathbf{x}) \leq 0 \Rightarrow$ $f_{2}(\mathbf{x}) \leq 0$ holds if and only if there exists a $\beta$ satisfying

$$
\beta\left[\begin{array}{cc}
\mathbf{Q}_{1} & \mathbf{p}_{1}^{H} \\
\mathbf{p}_{1} & e_{1}
\end{array}\right]-\left[\begin{array}{cc}
\mathbf{Q}_{2} & \mathbf{p}_{2}^{H} \\
\mathbf{p}_{2} & e_{2}
\end{array}\right] \geq \mathbf{0} .
$$

Next, we have

$$
\begin{aligned}
& \left|\left(\tilde{\mathbf{g}}_{m}[k]+\Delta \mathbf{g}_{m}[k]\right) \mathbf{\Xi}_{m}[k] \mathbf{v}_{m}[k]\right|^{2} \\
= & \left(\left(\tilde{\mathbf{g}}_{m}[k]+\Delta \mathbf{g}_{m}[k]\right) \boldsymbol{\Xi}_{m}[k] \mathbf{v}_{m}[k]\right)\left(\left(\tilde{\mathbf{g}}_{m}[k]+\Delta \mathbf{g}_{m}[k]\right) \boldsymbol{\Xi}_{m}[k] \mathbf{v}_{m}[k]\right)^{H} \\
= & \Delta \mathbf{g}_{m}[k] \mathbf{\Xi}_{m}[k] \mathbf{V}_{m}[k] \mathbf{\Xi}_{m}[k]^{H} \Delta \mathbf{g}_{m}[k]^{H} \\
& +2 \operatorname{Re}\left(\tilde{\mathbf{g}}_{m}[k] \boldsymbol{\Xi}_{m}[k] \mathbf{V}_{m}[k] \boldsymbol{\Xi}_{m}[k]^{H} \Delta \mathbf{g}_{m}[k]^{H}\right) \\
& +\tilde{\mathbf{g}}_{m}[k] \boldsymbol{\Xi}_{m}[k] \mathbf{V}_{m}[k] \boldsymbol{\Xi}_{m}[k]^{H} \tilde{\mathbf{g}}_{m}[k]^{H} .
\end{aligned}
$$

Similarly, $\sum_{j \neq m} \Upsilon_{m}[k]$ can be expressed as

$$
\begin{aligned}
& \sum_{j \neq m} \Upsilon_{m}[k]=\sum_{j \neq m}\left|\left(\tilde{\mathbf{g}}_{m}[k]+\Delta \mathbf{g}_{m}[k]\right) \boldsymbol{\Xi}_{m}[k] \mathbf{v}_{j}[k]\right|^{2} \\
= & \Delta \mathbf{g}_{m}[k] \boldsymbol{\Xi}_{m}[k]\left(\sum_{j \neq m} \mathbf{V}_{j}[k]\right) \boldsymbol{\Xi}_{m}[k]^{H} \Delta \mathbf{g}_{m}[k]^{H} \\
& +2 \operatorname{Re}\left(\tilde{\mathbf{g}}_{m}[k] \boldsymbol{\Xi}_{m}[k]\left(\sum_{j \neq m} \mathbf{V}_{j}[k]\right) \boldsymbol{\Xi}_{m}[k]^{H} \Delta \mathbf{g}_{m}[k]^{H}\right) \\
& +\tilde{\mathbf{g}}_{m}[k] \boldsymbol{\Xi}_{m}[k]\left(\sum_{j \neq m} \mathbf{V}_{j}[k]\right) \boldsymbol{\Xi}_{m}[k]^{H} \tilde{\mathbf{g}}_{m}[k]^{H} .
\end{aligned}
$$

Next, we transform (33b) into the following three constraints

$$
\begin{aligned}
& \mu_{m, k} \leq\left|\left(\tilde{\mathbf{g}}_{m}[k]+\Delta \mathbf{g}_{m}[k]\right) \boldsymbol{\Xi}_{m}[k] \mathbf{v}_{m}[k]\right|^{2}, \\
& \tau_{m, k} \theta_{m, k} \leq \mu_{m, k}, \\
& \theta_{m, k} \geq \sum_{j \neq m}^{M} \Upsilon_{m}[k]+B N_{0} / K .
\end{aligned}
$$

Combining (36), (33c) and Lemma 1, (38a) can be transformed into the following convex LMI constraint

$$
\left[\begin{array}{cc}
\beta_{m, k} \mathbf{I}+\hat{\mathbf{V}}_{m}[k] & \left(\tilde{\mathbf{g}}_{m}[k] \hat{\mathbf{V}}_{m}[k]\right)^{H} \\
\tilde{\mathbf{g}}_{m}[k] \hat{\mathbf{V}}_{m}[k] & \boldsymbol{\varepsilon}+\tilde{\mathbf{g}}_{m}[k] \hat{\mathbf{V}}_{m}[k] \tilde{\mathbf{g}}_{m}[k]^{H}-\mu_{m, k}
\end{array}\right] \geq \mathbf{0},
$$

where $\hat{\mathbf{V}}_{m}[k]=\boldsymbol{\Xi}_{m}[k] \mathbf{V}_{m}[k] \boldsymbol{\Xi}_{m}[k]^{H}$. The non-convex constraint (38b) can be expressed as the following convex constraint

$$
\frac{\tau_{m, k}^{(n)}}{2 \theta_{m, k}^{(n)}} \theta_{m, k}^{2}+\frac{\theta_{m, k}^{(n)}}{2 \tau_{m, k}^{(n)}} \tau_{m, k}^{2} \leq \mu_{m, k},
$$

where $\tau_{m, k}^{(n)}$ and $\theta_{m, k}^{(n)}$ are the values of $\tau_{m, k}$ and $\theta_{m, k}$ at the $n$th iteration, respectively. In addition, combining (37), (33c) and Lemma 1, (38c) can be transformed into the following convex LMI constraint

$$
\left[\begin{array}{cc}
\beta_{m, k} \mathbf{I}-\check{\mathbf{V}}_{m}[k] & -\left(\tilde{\mathbf{g}}_{m}[k] \check{\mathbf{V}}_{m}[k]\right)^{H} \\
-\tilde{\mathbf{g}}_{m}[k] \check{\mathbf{V}}_{m}[k] & \varepsilon-\tilde{\mathbf{g}}_{m}[k] \check{\mathbf{V}}_{m}[k] \tilde{\mathbf{g}}_{m}[k]^{H}+\theta_{m, k}-B N_{0} / K
\end{array}\right] \geq \mathbf{0}
$$

where $\check{\mathbf{V}}_{m}[k]=\boldsymbol{\Xi}_{m}[k]\left(\sum_{j \neq m} \mathbf{V}_{j}[k]\right) \boldsymbol{\Xi}_{m}[k]^{H}$.

Finally, we transform (28) into the following SDP problem

$$
\begin{aligned}
& \max _{\left\{\tau_{m, k}, \mu_{m, k}, \theta_{m, k},\left\{\mathbf{V}_{m}[k]\right\}\right\}} \sum_{m=1}^{M} \sum_{k=1}^{K} a_{m} \log \left(1+\tau_{m, k}\right) \\
& \text { s.t. }(39),(40),(41), \\
& \quad \sum_{M=1}^{M} \sum_{k=1}^{K} \operatorname{Tr}\left(\mathbf{F}^{H} \mathbf{F} \mathbf{V}_{m}[k]\right) \leq P_{\max }, \\
& \quad \operatorname{rank}\left(\mathbf{V}_{m}[k]\right)=1, \mathbf{V}_{m}[k] \geq 0 .
\end{aligned}
$$

It is obvious that (42) can be solved with the convex optimization toolbox such as CVX by removing the rank-one constraint. Likewise, the optimal digital beamforming matrix $\mathbf{V}_{m}[k]$ is obtained by iteratively solving problem (42).

To handle problem (42), we remove the rank-one constraint $\operatorname{rank}\left(\mathbf{V}_{m}[k]\right)=1$. If problem (42) does not yield a rank-one

(36) solution, namely $\operatorname{rank}\left(\mathbf{V}_{m}[k]\right)>1$, the Gaussian randomization technique is used to obtain a rank-one solution [34].

Now, we analyze the computational complexity for solving problem (42). Let $\epsilon$ be the iteration accuracy; then, the number of iterations is on the order of $\sqrt{3 K M N_{\mathrm{RF}}+7 K M} \ln (1 / \epsilon)$ [35]. For problem (42), there are $2 K M$ LMI constraints of size $N_{\text {IRS }}+1, K M$ LMI constraints of size $N_{\text {IRS }}, 3 K M+1$ LMI constraints of size 1, and $K M$ second-order cone constraints. Therefore, the complexity of solving problem (42) is on the order of $\sqrt{\Delta_{1}} \zeta \ln (1 / \epsilon)\left(\Delta_{2}+\zeta \Delta_{3}+\zeta^{2}\right)$, where $\Delta_{1}=$ $3 K M N_{\mathrm{RF}}+7 K M, \Delta_{2}=K M\left(2\left(N_{\mathrm{RF}}+1\right)^{3}+N_{\mathrm{RF}}^{3}+11\right)+1$, $\Delta_{3}=2 K M\left(N_{\mathrm{RF}}+1\right)^{2}+K M N_{\mathrm{RF}}^{2}+3 K M+1$, and $\zeta=K M N_{\mathrm{RF}}^{2}$. 


\section{B. Optimization of $\mathbf{\Phi}$ under Fixed $\mathbf{F}$ and $\mathbf{V}_{m}[k]$}

After obtaining the analog beamforming $\mathbf{F}$ and digital beamforming $\mathbf{v}_{m}[k]$, we reconsider the reflection matrix $\boldsymbol{\Phi}$ with imperfect CSIs. According to the definition in Section III-B, we have

$$
u_{m}[k] \mathbf{g}_{m}[k] \mathbf{\Phi H}[k] \mathbf{F} \mathbf{v}_{m}[k] \triangleq \mathbf{g}_{m}[k] \mathbf{\Phi} \mathbf{z}_{m}[k] .
$$

Next, we define $\mathbf{Z}_{m}[k] \triangleq \operatorname{diag}\left(\mathbf{z}_{m}[k]\right)$, and thus, we have $\mathbf{g}_{m}[k] \mathbf{\Phi} \mathbf{z}_{m}[k]=\mathbf{g}_{m}[k] \mathbf{Z}_{m}[k] \phi$. After that, the achievable rate can be expressed as

$$
R_{m}[k]=\frac{B}{K} \log \left(1+\frac{\left|\left(\tilde{\mathbf{g}}_{m}[k]+\Delta \mathbf{g}_{m}[k]\right) \mathbf{Z}_{m}[k] \boldsymbol{\phi}\right|^{2}}{\sum_{j \neq m}^{M} \Upsilon_{m}^{\prime}[k]+B N_{0} / K}\right),
$$

where $\Upsilon_{m}^{\prime}[k]=\left|\left(\tilde{\mathbf{g}}_{m}[k]+\Delta \mathbf{g}_{m}[k]\right) \mathbf{Z}_{j}[k] \phi\right|^{2}$. It can be found that (44) has a similar expression with (32), and thus, the same scheme can be used to solve the reflection matrix $\boldsymbol{\Phi}$. Therefore, we omit the details and directly formulate the following SDR problem as

$$
\begin{aligned}
& \max _{\left\{\tau_{m, k}^{\prime}, \mu_{m, k}^{\prime}, \theta_{m, k}^{\prime}, \mathbf{\Omega}\right\}} \sum_{m=1}^{M} \sum_{k=1}^{K} a_{m} \log \left(1+\tau_{m, k}^{\prime}\right) \\
& \text { s.t. }\left[\begin{array}{cc}
\beta_{m, k}^{\prime} \mathbf{I}+\hat{\mathbf{\Omega}}_{m}[k] & \left(\tilde{\mathbf{g}}_{m}[k] \hat{\mathbf{\Omega}}_{m}[k]\right)^{H} \\
\tilde{\mathbf{g}}_{m}[k] \hat{\mathbf{\Omega}}_{m}[k] & \boldsymbol{\varepsilon}+\tilde{\mathbf{g}}_{m}[k] \hat{\mathbf{\Omega}}_{m}[k] \tilde{\mathbf{g}}_{m}[k]^{H}-\mu_{m, k}^{\prime}
\end{array}\right] \geq \mathbf{0}, \\
& \frac{\tau_{m, k}^{\prime(n)}}{2{\theta^{\prime}}_{m, k}^{(n)}} \theta_{m, k}^{\prime 2}+\frac{{\theta_{m, k}^{\prime}}_{m, k}^{(n)}}{2{\tau_{m, k}^{\prime}(n)}_{m, k}^{\prime 2}} \leq \mu_{m, k}^{\prime} \\
& {\left[\begin{array}{cc}
\beta_{m, k}^{\prime} \mathbf{I}-\check{\mathbf{\Omega}}_{m}[k] & -\left(\tilde{\mathbf{g}}_{m}[k] \check{\mathbf{\Omega}}_{m}[k]\right)^{H} \\
-\widetilde{\mathbf{g}}_{m}[k] \check{\mathbf{\Omega}}_{m}[k] & \boldsymbol{\varepsilon}-\tilde{\mathbf{g}}_{m}[k] \check{\mathbf{\Omega}}_{m}[k] \tilde{\mathbf{g}}_{m}[k]^{H}+\theta_{m, k}^{\prime}-B N_{0} / K
\end{array}\right] \geq \mathbf{0},}
\end{aligned}
$$

$$
\boldsymbol{\Omega}(i, i)=1, \mathbf{\Omega} \geq 0,
$$

where $\tau_{m, k}, \mu_{m, k}^{\prime}$ and $\theta_{m, k}^{\prime}$ are the introduced auxiliary variables, $\hat{\boldsymbol{\Omega}}_{m}[k]=\mathbf{Z}_{m}[k] \boldsymbol{\Omega} \mathbf{Z}_{m}[k]^{H}$, and $\check{\boldsymbol{\Omega}}_{m}[k]=\sum_{j \neq m} \boldsymbol{\Xi}_{j}[k] \boldsymbol{\Omega} \boldsymbol{\Xi}_{j}[k]^{H}$. We can use the CVX toolbox to solve (45). Likewise, if problem (29) does not yield a rank-one solution, namely $\operatorname{rank}\left(\mathbf{\Omega}^{\star}\right) \neq 1$. The Gaussian randomization technique can be used to obtain a rank-one solution [34].

Now, we analyze the computational complexity for solving problem (45). Let $\epsilon$ be the iteration accuracy; then, the number of iterations is on the order of $\sqrt{K M N_{\text {IRS }}+7 K M} \ln (1 / \epsilon)$. For problem (45), there are $2 K M$ LMI constraints of size $N_{\text {IRS }}+1,1$ LMI constraint of size $N_{\text {IRS }}, 4 K M$ LMI constraints of size 1 , and $K M$ second-order cone constraints. Therefore, the complexity of solving problem (45) is on the order of $\sqrt{\Delta_{1}} \zeta \ln [1 / \epsilon)\left(\Delta_{2}+12 K M+1+\zeta \Delta_{2}+\zeta^{2}\right]$, where $\Delta_{1}=K M N_{\text {IRS }}+7 K M, \Delta_{2}=2 K M\left(N_{\text {IRS }}+1\right)^{3}+N_{\text {IRS }}^{3}+13 K M$, $\Delta_{3}=2 K M\left(N_{\mathrm{RF}}+1\right)^{2}+N_{\mathrm{RF}}^{2}+4 K M$, and $\zeta=N_{I R S}^{2}$.

\section{Numerical Results}

In this section, simulation results are presented to evaluate the performance of the proposed schemes in IRS-aided $\mathrm{THz}$ MIMO-OFDMA systems. Due to the severe pathloss in $\mathrm{THz}$, we consider a short distance communication scenario as shown in Fig. 3, where users are located within a circle with $1.5 \mathrm{~m}$ radius. The AoD/AOA follows the uniform distribution within

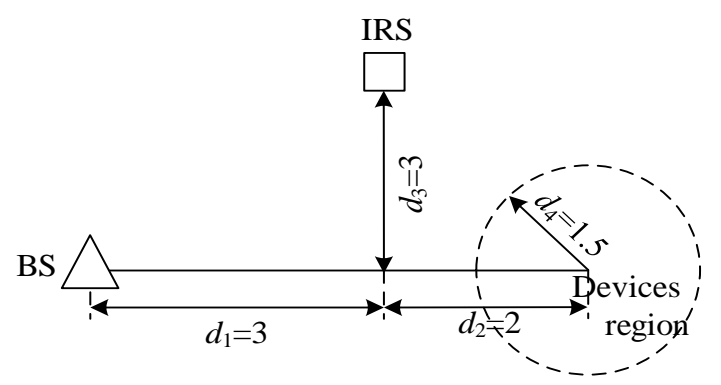

Fig. 3: The location distribution in the IRS-aided system.

TABLE I: Default Parameters Used in Simulations.

\begin{tabular}{l|r}
\hline Parameters & Value \\
\hline \hline Number of antennas & $N_{\mathrm{TX}}=64$ \\
\hline Number of RF chains & $N_{\mathrm{RF}}=4$ \\
\hline Number of reflection elements & $N_{\mathrm{IRS}}=4$ \\
\hline Central frequency & $f_{c}=340[\mathrm{GHz}]$ \\
\hline Bandwidth & $B=20[\mathrm{GHz}]$ \\
\hline Number of subcarriers & $K=16$ \\
\hline Number of users & $M=2$ \\
\hline Transmit antenna gain & $G_{t}=4+20 \log _{10}\left(\sqrt{N_{\mathrm{TX}}}\right)$ \\
\hline Receive antenna gain & $G_{r}=1$ \\
\hline Absorption coefficient & $0.0033 / m$ \\
\hline Speed of light & $c=3 \times 10^{8}$ \\
\hline Noise variance & $N_{0}=-174[\mathrm{dBm} / \mathrm{Hz}]$ \\
\hline
\end{tabular}

$[-\pi / 2, \pi / 2]$, and the antenna spacing is assumed to be half wavelength. The default simulation parameters are listed in Table I, and they are used in simulation unless otherwise specified.

Figs. 4 and 5 show the convergence performance of the proposed inner iterative algorithm for solving the digital beamforming and reflection matrix, respectively, i.e., Line $5 \sim 9$ and Line $11 \sim 15$ in Algorithm 1. Here, we set $\varepsilon=0, P_{\max }=0$ $\mathrm{dBm}$ and $\alpha_{m}=1(m \in\{1,2\})$. The legend " $n$th iteration" in Figs. 4 and 5 stands for the outer iteration number. One can observe that the inner iterative algorithm tends to converge after 5 iterations for each outer iteration. In addition, it can be found that the gap is small between the 2nd and 3rd iterations, but large between the 1 st and 2 nd iteration. This means that outer iterative loop (i.e., Algorithm 1) also converges rapidly, and we will elaborate this in detail in Fig. 6.

The convergence performance of Algorithm 1 under different estimation errors is plotted in Fig. 6, where we set the maximum transmit power $P_{\max }=4 \mathrm{dBm}$ and $\alpha_{m}=1(m \in\{1,2\})$. It is clear that the rate tends to stabilize after 3 iterations, which demonstrates the fast convergence of the proposed algorithm. In addition, it is easy to understand that the rate is low for a large estimation error as shown in Fig. 6, where $\varepsilon=0$ means perfect CSIs between the IRS and users.

Fig. 7 shows the rate versus $P_{\max }$ under different estimation errors, where we set $\alpha_{m}=1(m \in\{1,2\})$. Meanwhile, we also plot the rate under the fully digital structure, namely each antenna is connected to each RF chain. It is clear that the rate under the fully digital structure is higher than that under the sparse RF chain structure for the same condition, while the circuit power consumption is very high for the former. This is also one of the reasons for which the sparse RF chain structure 


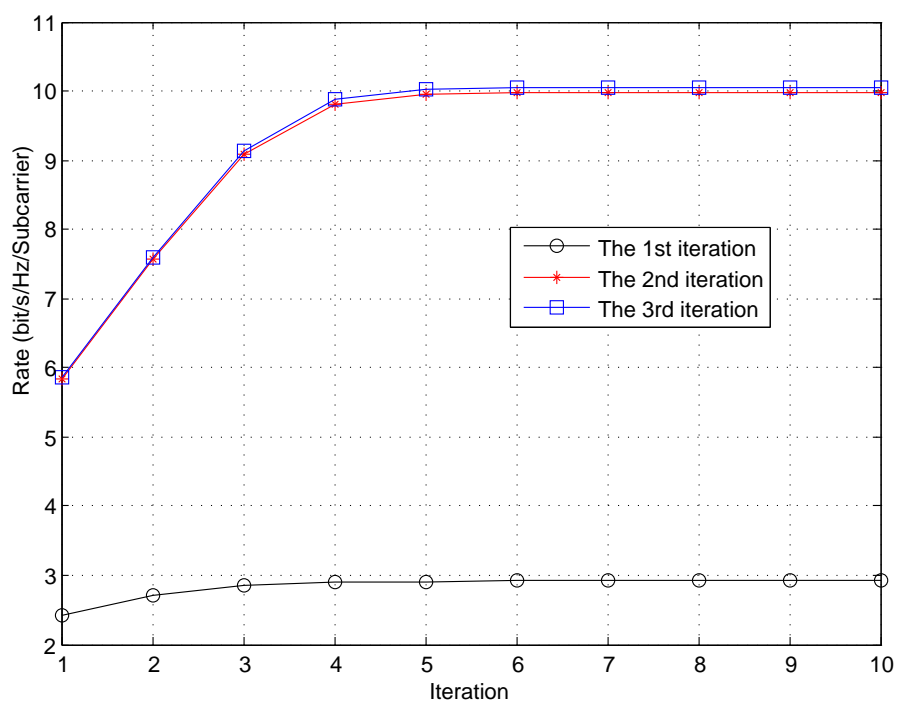

Fig. 4: The rate versus iteration for solving the digital beamforming.

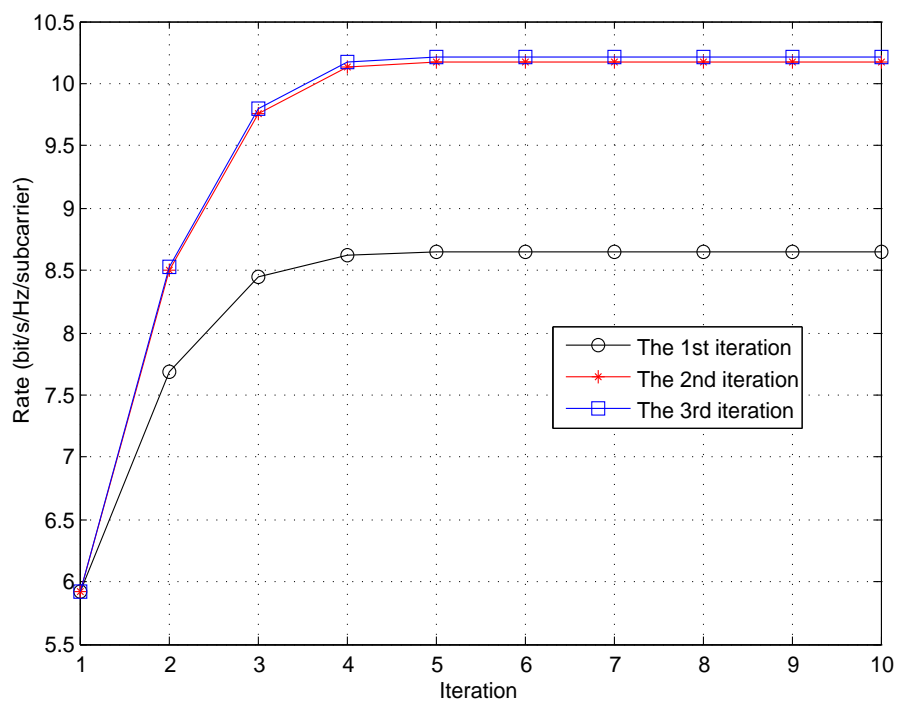

Fig. 5: The rate versus iteration for solving the reflection matrix.

is usually adopted when the ultra high frequency carrier is applied. In addition, one can observe that the rate increases with $P_{\max }$.

We plot the rate under different BS antennas in Fig. 8, where we set $P_{\text {max }}=4 \mathrm{dBm}$ and $\alpha_{m}=1(m \in\{1,2\})$. It is obvious that the rate increases with the number of antennas, but with a decreasing slope. In addition, we also plot the rate versus $P_{\text {max }}$ for different numbers of IRS reflection elements in Fig. 9, with $\varepsilon=0$. It is observed again that a large number of IRS reflection elements leads to a higher rate. This is because a higher beamforming gain can be achieved when there are either more antennas at the BS or more reflection elements at the IRS.

To compare the system performance under different users' weights, we set the user weight $\alpha=\left[\begin{array}{ll}1 & 1\end{array}\right]$ and $\alpha=\left[\begin{array}{ll}1 & 3\end{array}\right]$ as shown in Figs. 10 and 11 . Under $\alpha=\left[\begin{array}{ll}1 & 1\end{array}\right]$, it is clear the

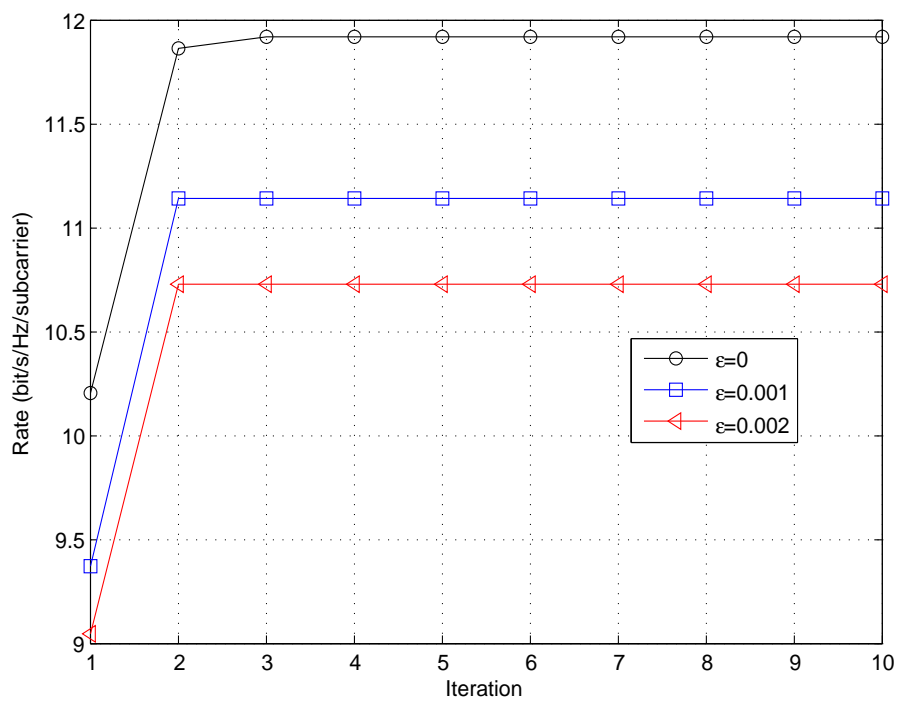

Fig. 6: The rate versus iteration for the proposed Algorithm 1.

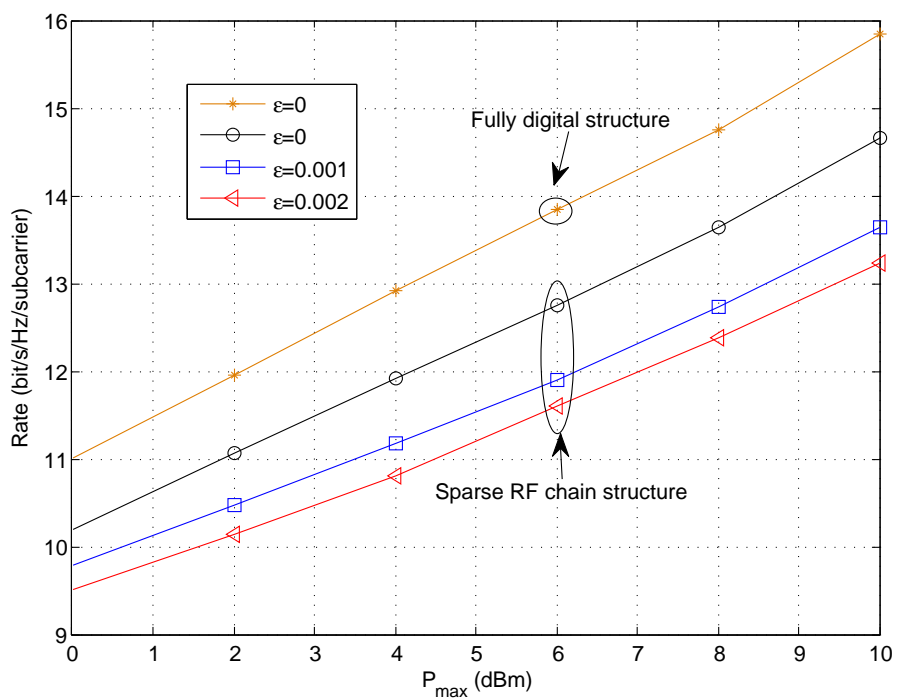

Fig. 7: The rate versus the allowable maximum transmit power.

objective is to just maximize the sum rate. This explains why the sum rate with $\alpha=\left[\begin{array}{ll}1 & 3\end{array}\right]$ is always lower than that with $\alpha=\left[\begin{array}{ll}1 & 1\end{array}\right]$. If we look at the individual rates for the two users, the rate for user 1 decreases, while that for user 2 increases when changing from $\alpha=\left[\begin{array}{ll}1 & 1\end{array}\right]$ to $\alpha=\left[\begin{array}{ll}1 & 3\end{array}\right]$. This is expected, since a higher priority is given to user 2 . Moreover, the sum rate declines while the system fairness increases. In practice, we can set different weights according to different quality of service requirements of the users.

\section{Conclusion}

In this paper, we have considered an IRS-aided THz MIMOOFDMA system, where the BS is equipped with a sparse RF chain structure. First, we have proposed a joint hybrid analog/digital beamforming and reflection matrix design to maximize the weighted sum rate under perfect CSIs. Next, 


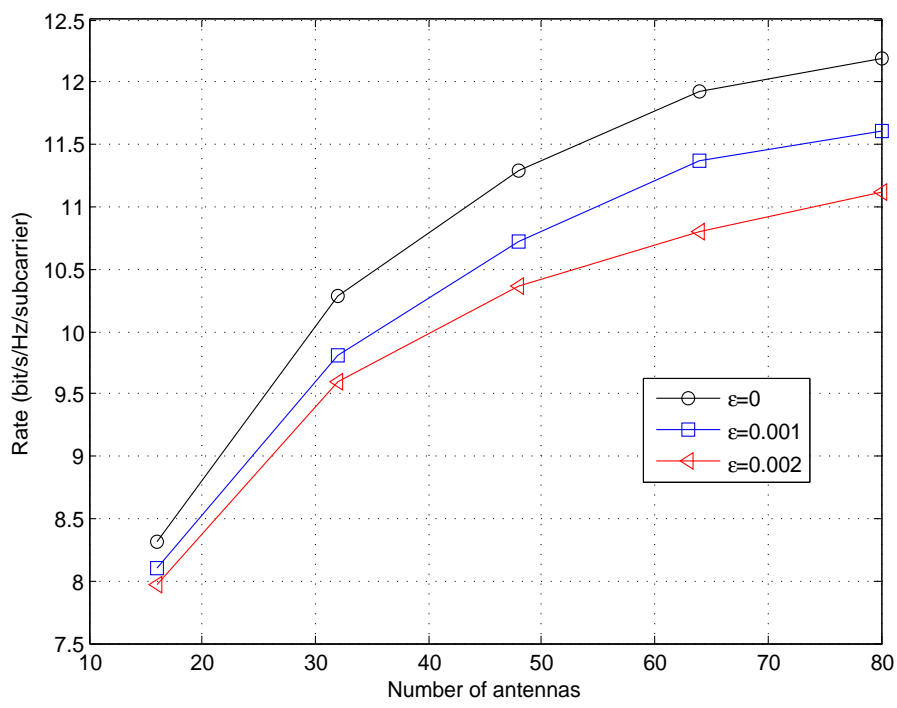

Fig. 8: The rate versus the number of antennas.

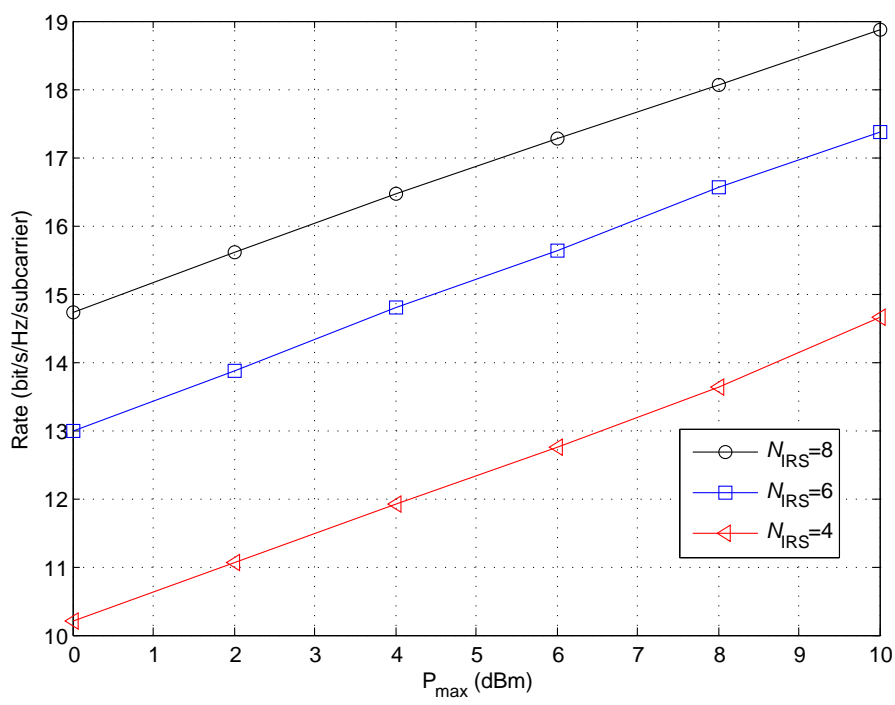

Fig. 9: The rate versus the allowable maximum transmit power under different numbers of IRS reflection elements.

considering the imperfect CSIs from the IRS to users, we have redesigned a robust joint optimization algorithm. From simulation results, we have found that the channel estimation error has a large impact on the system sum rate. Moreover, allocating a higher weight to a particular user can improve that user's rate, but at the cost of sum rate. Consequently, channel estimation schemes and users weight selection are important criteria for the design of practical systems, and we need to adjust the weights according to different quality of service requirements of the users.

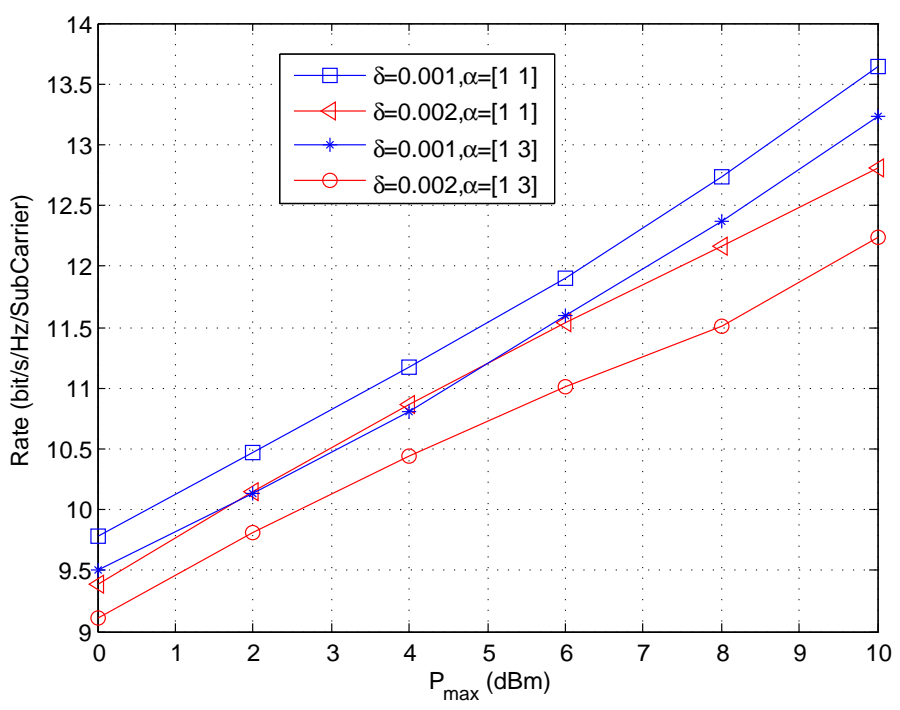

Fig. 10: The rate versus the allowable maximum transmit power with different weights.

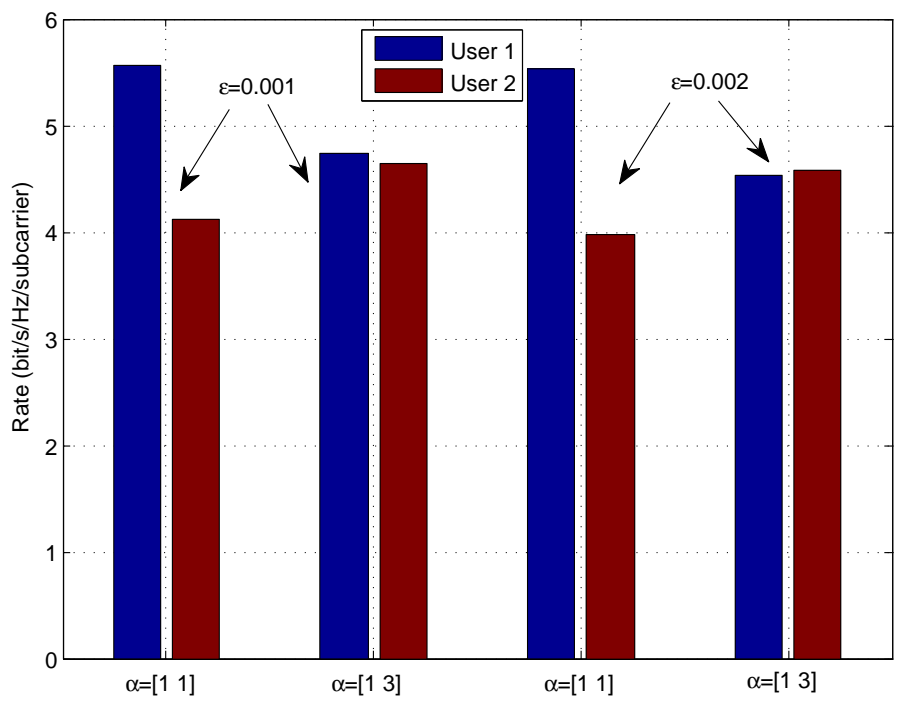

Fig. 11: Rate comparison under different weights.

\section{Appendix A}

Proof of Theorem 1

First, we give the Lagrangian function of (25) without the rank-one constraint as

$$
F\left(t_{m, k}, b_{m, k}, \xi, \psi_{m, k}, v_{m, k}, \mathbf{V}_{m}[k]\right)=\sum_{m=1}^{M} \sum_{k=1}^{K} a_{m} \log \left(1+t_{m, k}\right)
$$

$+\xi\left(P_{\max }-\sum_{M=1}^{M} \sum_{k=1}^{K} \operatorname{Tr}\left(\mathbf{F}^{H} \mathbf{F} \mathbf{V}_{m}[k]\right)\right)$

$+\sum_{m=1}^{M} \sum_{k=1}^{K} \psi_{m, k}\left(b_{m, k}-\left(\sum_{j \neq m}^{M} \operatorname{Tr}\left(\overline{\mathbf{H}}_{m}[k] \mathbf{V}_{j}[k]\right)+\delta^{2}\right)\right)$

$+\sum_{m=1}^{M} \sum_{k=1}^{K} v_{m, k}\left(\operatorname{Tr}\left(\overline{\mathbf{H}}_{m}[k] \mathbf{V}_{m}[k]\right)-\left(\frac{t_{m, k}^{(n)}}{2 b_{m, k}^{(n)}} b_{m, k}^{2}+\frac{b_{m, k}^{(n)}}{2 t_{m, k}^{(n)}} t_{m, k}^{2}\right)\right)$

$+\sum_{m=1}^{M} \sum_{k=1}^{K} \operatorname{Tr}\left(\Theta_{m, k} \mathbf{V}_{m}[k]\right)$. 
where $\xi, \psi_{m, k}, v_{m, k}, \Theta_{m, k}$, respectively, represent the Lagrange multipliers corresponding the constraints (21c), (22b), (24) and (25c). Since the relaxed SDP problem (25) is convex, and the gap between the primal problem and its dual problem is zero, namely it satisfies the Slater's condition [37]. Therefore, the Karaush-Kuhn-Tucker (KKT) conditions are necessary and sufficient for the optimal solutions of problem (25) with rankone constraint. Next, we give the KKT conditions related to the optimal digital beamforming $\mathbf{V}_{m}[k]^{\star}$ as:

$$
\begin{aligned}
\xi^{\star} \mathbf{F}^{H} \mathbf{F}+\sum_{j \neq m}^{M} \sum_{k=1}^{K} \psi_{m, k}^{\star} \overline{\mathbf{H}}_{j}[k]-v_{m, k}^{\star} \overline{\mathbf{H}}_{m}[k] & =\boldsymbol{\Theta}_{m, k}^{\star}, \\
\boldsymbol{\Theta}_{m, k}^{\star} \mathbf{V}_{m}[k]^{\star} & =\mathbf{0}, \\
\boldsymbol{\Theta}_{m, k}^{\star} & \geq \mathbf{0},
\end{aligned}
$$

where $\xi^{\star}, \psi_{m, k}^{\star}, v_{m, k}^{\star}$ and $\boldsymbol{\Theta}_{m, k}^{\star}$ are the optimal Lagrange multipliers. The analog beamforming can be expressed as $\mathbf{F}=\left[\mathbf{f}_{1}, \cdots, \mathbf{f}_{\mathrm{RF}}\right]$, and we have $\mathbf{f}_{i}^{H} \mathbf{f}_{i}=1$ and $\mathbf{f}_{i}^{H} \mathbf{f}_{j} \ll 1(i \neq j)$ for a large $N_{\mathrm{TX}}$ as our analysis for the analog beamforming matrix in Section III-A. In this way, for a large $N_{\mathrm{TX}}$ and i.i.d. channels, we can obtain $\mathbf{F}^{H} \mathbf{F} \approx \mathbf{I}$, and $\mathbf{F}^{H} \mathbf{F}$ is a full rank matrix, namely $\operatorname{rank}\left(\mathbf{F}^{H} \mathbf{F}\right)=N_{\mathrm{RF}}$. Because $\xi^{\star}>0, \psi_{m, k}^{\star}>0$, we define

$$
\mathbf{Y}=\xi^{\star} \mathbf{F}^{H} \mathbf{F}+\sum_{j \neq m}^{M} \sum_{k=1}^{K} \psi_{m, k}^{\star} \overline{\mathbf{H}}_{j}[k]
$$

and thus, $\mathbf{Y}$ is a positive-definite matrix which has full rank with $\operatorname{rank}(\mathbf{Y})=N_{\mathrm{RF}}$. Based on this, we have

$$
\begin{aligned}
\operatorname{rank}\left(\boldsymbol{\Theta}_{m, k}^{\star}\right) & =\operatorname{rank}\left(\mathbf{Y}-v_{m, k}^{\star} \overline{\mathbf{H}}_{m}[k]\right) \\
& \geq \operatorname{rank}(\mathbf{Y})-\operatorname{rank}\left(v_{m, k}^{\star} \overline{\mathbf{h}}_{m}[k]^{H} \overline{\mathbf{h}}_{m}[k]\right) \\
& \geq N_{\mathrm{RF}}-1 .
\end{aligned}
$$

Therefore, we can claim that the rank of $\Theta_{m, k}^{\star}$ is either $N_{\mathrm{RF}}$ or $N_{\mathrm{RF}}-1$. If $\operatorname{rank}\left(\boldsymbol{\Theta}_{m, k}^{\star}\right)=N_{\mathrm{RF}}$, according to $(47 \mathrm{~b})$, the optimal $\mathbf{V}_{m}[k]^{\star}=\mathbf{0}$, which means that the BS does not transmit any signal. Thus, we have $\operatorname{rank}\left(\Theta_{m, k}^{\star}\right)=N_{\mathrm{RF}}-1$, and the null space of $\boldsymbol{\Theta}_{m, k}^{\star}$ is one dimensional. Meanwhile, (47b) means that $\mathbf{V}_{m}[k]^{\star}$ must lie in the null-space of $\boldsymbol{\Theta}_{m, k}^{\star}$, and we have $\operatorname{rank}\left(\mathbf{V}_{m}[k]^{\star}\right)=1$ and the proof is completed.

\section{REFERENCES}

[1] Z. Piao, M. Peng, Y. Liu, and M. Daneshmand, "Recent advances of edge cache in radio access networks for internet of things: Techniques, performances, and challenges," IEEE Internet Things J., vol. 6, no. 1, pp. 1010-1028, Feb. 2019.

[2] G. Yu, X. Chen, C. Zhong, D. W. Kwan Ng, and Z. Zhang, "Design, analysis, and optimization of a large intelligent reflecting surface-aided B5G cellular internet of things," IEEE Internet Things J., vol. 7, no. 9, pp. 8902-8916, Sep. 2020

[3] H. Sarieddeen, M. Alouini, and T. Y. Al-Naffouri, "Terahertz-band ultramassive spatial modulation MIMO,' IEEE J. Sel. Areas Commun., vol. 37, no. 9 , pp. 2040-2052, Sep. 2019.

[4] R. Zhang, W. Hao, G. Sun, and S. Yang, "Hybrid precoding design for wideband THz massive MIMO-OFDM systems with beam squint," IEEE Sys. J., pp. 1-4, to be published, 2020.

[5] S. Priebe and T. Kurner, "Stochastic modeling of $\mathrm{THz}$ indoor radio channels," IEEE Trans. Wireless Commun., vol. 12, no. 9, pp. 4445-4455, Sep. 2013.

[6] L. Lu, G. Y. Li, A. L. Swindlehurst, A. Ashikhmin, and R. Zhang, "An overview of massive mimo: Benefits and challenges," IEEE J. Sel. Topics Signal Proc., vol. 8, no. 5, pp. 742-758, May 2014.
[7] M. Zeng, X. Li, G. Li, W. Hao, and O. A. Dobre, "Sum rate maximization for IRS-assisted uplink NOMA," IEEE Commun. Lett., vol. 25, no. 1, pp. 234-238, Jan. 2021.

[8] J. Zhang, E. Björnson, M. Matthaiou, D. W. K. Ng, H. Yang, and D. J. Love, "Prospective multiple antenna technologies for beyond 5G," IEEE J. Sel. Areas Commun., vol. 38, no. 8, pp. 1637-1660, Aug. 2020.

[9] Q. Wu and R. Zhang, "Intelligent reflecting surface enhanced wireless network via joint active and passive beamforming," IEEE Trans. Wireless Commun., vol. 18, no. 11, pp. 5394-5409, Nov. 2019.

[10] Z. Chu, W. Hao, P. Xiao, and J. Shi, "Intelligent reflecting surface aided multi-antenna secure transmission," IEEE Wireless Commun. Lett., vol. 9, no. 1, pp. 108-112, Jan. 2020.

[11] Y. Chen, Y. Wang, J. Zhang, and Z. Li, "Resource allocation for intelligent reflecting surface aided vehicular communications," IEEE Trans. Veh. Tech., vol. 69, no. 10, pp. 12321-12326, Oct. 2020.

[12] W. Hao, G. Sun, J. Zhang, P. Xiao, and L. Hanzo, "Secure millimeter wave cloud radio access networks relying on microwave multicast fronthaul," IEEE Trans. Commun., vol. 68, no. 5, pp. 3079-3095, May 2020.

[13] C. Lin and G. Y. Li, "Adaptive beamforming with resource allocation for distance-aware multi-user indoor terahertz communications," IEEE Trans. Wireless Commun., vol. 63, no. 8, pp. 2985-2995, Aug. 2015.

[14] — , "Energy-efficient design of indoor mmwave and sub-THz systems with antenna arrays," IEEE Trans. Wireless Commun., vol. 15, no. 7, pp. 4660-4672, Jul. 2016.

[15] S. A. Busari, K. M. S. Huq, S. Mumtaz, J. Rodriguez, Y. Fang, D. C. Sicker, S. Al-Rubaye, and A. Tsourdos, "Generalized hybrid beamforming for vehicular connectivity using THz massive MIMO," IEEE Trans. Veh. Tech., vol. 68, no. 9, pp. 8372-8383, Sep. 2019.

[16] J. Tan and L. Dai, "Delay-phase precoding for THz massive MIMO with beam split," in Proc. IEEE GLOBECOM, 2019, pp. 1-6.

[17] H. Yuan, N. Yang, K. Yang, C. Han, and J. An, "Hybrid beamforming for terahertz MIMO-OFDM systems over frequency selective fading," arXiv e-prints, p. arXiv: 1910.05967, 2019

[18] B. Ning, Z. Chen, W. Chen, Y. Du, and J. Fang, "Terahertz multi-user massive MIMO with intelligent reflecting surface: Beam training and hybrid beamforming," IEEE Trans. Veh. Technol., to be published, 2021.

[19] S. Zhang and R. Zhang, "Capacity characterization for intelligent reflecting surface aided MIMO communication," IEEE J. Sel. Areas Commun., vol. 38, no. 8, pp. 1823-1838, Aug. 2020

[20] Y. Yang, B. Zheng, S. Zhang, and R. Zhang, "Intelligent reflecting surface meets OFDM: Protocol design and rate maximization," IEEE Trans. Commun., vol. 68, no. 7, pp. 4522-4535, Jul. 2020.

[21] Z. Zhang and L. Dai, "A joint precoding framework for wideband reconfigurable intelligent surface-aided cell-free network," arXiv e-prints, p. arXiv:2002.03744, 2020.

[22] G. Zhou, C. Pan, H. Ren, K. Wang, M. Di Renzo, and A. Nallanathan, "Robust beamforming design for intelligent reflecting surface aided MISO communication systems," IEEE Wireless Commun. Lett., vol. 9, no. 10, pp. 1658-1662, Oct. 2020.

[23] Z. Wan, Z. Gao, and M. Alouini, "Broadband channel estimation for intelligent reflecting surface aided mmWave massive MIMO systems," in IEEE ICC, 2020, pp. 1-6.

[24] B. Zheng, C. You, and R. Zhang, "Intelligent reflecting surface assisted multi-user OFDMA: Channel estimation and training design," IEEE Trans. Wireless Commun., vol. 19, no. 12, pp. 8315-8329, Dec. 2020.

[25] Y. Yang, B. Zheng, S. Zhang, and R. Zhang, "Intelligent reflecting surface meets OFDM: Protocol design and rate maximization," IEEE Trans. Commun., vol. 68, no. 7, pp. 4522-4535, Jul. 2020.

[26] S. Priebe, M. Kannicht, M. Jacob, and T. Kürner, "Ultra broadband indoor channel measurements and calibrated ray tracing propagation modeling at THz frequencies," J. Commun. and Net., vol. 15, no. 6, pp. 547-558, Jun. 2013.

[27] W. Tang, M. Z. Chen, X. Chen, J. Y. Dai, Y. Han, M. Di Renzo, Y. Zeng, S. Jin, Q. Cheng, and T. J. Cui, "Wireless communications with reconfigurable intelligent surface: Path loss modeling and experimental measurement," IEEE Trans. Wireless Commun., vol. 20, no. 1, pp. 421439, Jan. 2021

[28] Z. Wei, Y. Cai, Z. Sun, D. W. K. Ng, J. Yuan, M. Zhou, and L. Sun, "Sum-rate maximization for IRS-assisted UAV OFDMA communication systems," IEEE Trans. Wireless Commun., pp. 1-1, 2020.

[29] S. Park, A. Alkhateeb, and R. W. Heath, "Dynamic subarrays for hybrid precoding in wideband mmwave MIMO systems," IEEE Trans. Wireless Commun., vol. 16, no. 5, pp. 2907-2920, May 2017.

[30] W. Shen, X. Bu, X. Gao, C. Xing, and L. Hanzo, "Beamspace precoding and beam selection for wideband millimeter-wave MIMO relying on lens 
antenna arrays," IEEE Trans. Signal Process., vol. 67, no. 24, pp. 63016313, Dec. 2019.

[31] P. Song, G. Scutari, F. Facchinei, and L. Lampariello, "D3m: Distributed multi-cell multigroup multicasting," in Proc. IEEE International Conference on Acoustics, Speech and Signal Processing (ICASSP), 2016, pp. 3741-3745.

[32] Q. Zhang, Q. Li, and J. Qin, "Robust beamforming for nonorthogonal multiple-access systems in MISO channels," IEEE Trans. Veh. Technol., vol. 65, no. 12, pp. 10231-10236, Dec. 2016.

[33] Z. Yang, C. Pan, W. Xu, Y. Pan, M. Chen, and M. Elkashlan, "Power control for multi-cell networks with non-orthogonal multiple access," IEEE Trans. Wireless Commun., vol. 17, no. 2, pp. 927-942, Feb. 2018.

[34] Z. Luo, W. Ma, A. M. So, Y. Ye, and S. Zhang, "Semidefinite relaxation of quadratic optimization problems," IEEE Signal Process. Mag., vol. 27, no. 3, pp. 20-34, Mar. 2010.

[35] K. Wang, A. M. So, T. Chang, W. Ma, and C. Chi, "Outage constrained robust transmit optimization for multiuser MISO downlinks: Tractable approximations by conic optimization," IEEE Trans. Signal Process., vol. 62, no. 21, pp. 5690-5705, Nov. 2014.

[36] F. Zhou, Z. Li, J. Cheng, Q. Li, and J. Si, "Robust AN-aided beamforming and power splitting design for secure MISO cognitive radio with SWIPT," IEEE Trans. Wireless Commun., vol. 16, no. 4, pp. 2450-2464, Apr. 2017.

[37] S. Boyd and L. Vandenberghe, "Convex optimization," Cambridge University Press, 2004. 